\title{
Combined MEK and PI3K/p110 $\beta$ inhibition as a novel targeted therapy for malignant mesothelioma displaying sarcomatoid features
}

Miriam Marqués ${ }^{1,2}$, Robin Tranchant ${ }^{3, *}$, Blanca Risa-Ebrí ${ }^{1}$, María L. Suárez-Solís ${ }^{1,4}$, Luis C. Fernández

1, Enrique Carrillo-de-Santa-Pau ${ }^{1,5}$, Natalia del Pozo ${ }^{1,2}$, Jaime Martínez de Villarreal ${ }^{1,2}$, Clement Meiller $^{3}$, Yves Allory ${ }^{1,6}$, Yuna Blum ${ }^{7}$, Christine Pirker ${ }^{8}$, Balazs Hegedus ${ }^{9}$, Simon T. Barry ${ }^{10}$, Amancio Carnero $^{1,2,11}$, Walter Berger ${ }^{8}$, Didier Jean ${ }^{3}$, Francisco X. Real ${ }^{1,2,12}$

${ }^{1}$ Epithelial Carcinogenesis Group, Spanish National Cancer Centre-CNIO, Madrid, Spain

${ }^{2}$ CIBERONC, Madrid, Spain

${ }^{3}$ Centre de Recherche des Cordeliers, INSERM, Université Paris Descartes, Université Paris Diderot, Sorbonne Université, USPC, Functional Genomics of Solid Tumors team, F-75006 Paris, France.

${ }^{4}$ Department of Surgical Pathology, Hospital Clínico San Carlos, Madrid, Spain.

${ }^{5}$ Computational Biology Group, Precision Nutrition and Cancer Research Program. IMDEA Food Institute. Madrid, Spain

${ }^{6}$ Université Paris-Est Créteil, France INSERM, U955, Institut Mondor de Recherche Biomédicales APHP, Hôpital Henri Mondor, Department of Pathology, Créteil, France

${ }^{7}$ Programme Cartes d'Identité des Tumeurs (CIT), Ligue Nationale Contre Le Cancer, Paris, France

${ }^{8}$ Institute of Cancer Research and Comprehensive Cancer Center, Medical University Vienna, Austria

${ }^{9}$ Department of Thoracic Surgery, Medical University Vienna, Austria

${ }^{10}$ IMED Oncology, AstraZeneca, Li Ka Shing Centre, Cambridge

${ }^{11}$ Instituto de Biomedicina de Sevilla, IBIS/HUVR/ Universidad de Sevilla/ Consejo Superior de Investigaciones Científicas; Avda. Manuel Siurot s/n 41013, Sevilla, Spain

${ }^{12}$ Departament de Ciències Experimentals i de la Salut, Universitat Pompeu Fabra, Barcelona, Spain

* Present address: Laboratoire de Biochimie (LBC), ESPCI Paris, PSL Research University, CNRS UMR8231, Chimie Biologie Innovation, Paris, France.

Keywords: sarcomatoid mesothelioma, PI3K, MAPK, GPCRs 


\section{Running title: Targeted therapies for sarcomatoid malignant mesothelioma}

Financial Support: This work was supported, in part, by grants from Asociación Española Contra el Cáncer (FXR), Spanish Ministry of Economy and Competitivity, Plan Estatal de I+D+I 2013-2016, ISCIII (FIS PI15/00045) (AC), RTICC (Instituto de Salud Carlos III, grants RD12/0036/0034 to FXR and AC, respectively), and CIBERONC (CB16/12/00453 and CD16/12/00275 to FXR and AC, respectively), cofunded by FEDER from Regional Development European Funds (European Union) and Inserm (Institut national de la santé et de la recherche médicale). MM was supported by a Sara Borrell Fellowship from Instituto de Salud Carlos III. CNIO is supported by Ministerio de Ciencia, Innovación y Universidades as a Centro de Excelencia Severo Ochoa SEV-2015-0510.

Corresponding authors: Francisco X. Real and Miriam Marqués, Epithelial Carcinogenesis Group, Spanish National Cancer Research Centre-CNIO, Madrid, 28029, Spain. E-mails: freal@cnio.es, mmarques@cnio.es

Competing interests: The authors state that they have no conflict of interest to disclose.

Word count main text: 5019

Figures: 7 main Figures, 7 Supplementary Figures.

Statement of significance: Mesothelioma is highly aggressive; its sarcomatoid variants have worse prognosis. Building on a genetic mouse model, a novel combination therapy is uncovered that is relevant to human tumors. 


\begin{abstract}
Among malignant mesotheliomas (MM) the sarcomatoid subtype is associated with higher chemoresistance and worst survival. Due to its low incidence, there has been little progress in the knowledge of the molecular mechanisms associated with sarcomatoid MM, which might help to define novel therapeutic targets. In this work, we show that loss of PTEN expression is frequent in human sarcomatoid MM and PTEN expression levels are lower in sarcomatoid MM than in the biphasic and epithelioid subtypes. We confirm that combined Pten and Trp53 deletion in mouse mesothelium leads to non-epithelioid MM development. In Pten;Trp53-null mice developing $\mathrm{MM}$, the Gai2 coupled receptor subunit drives MEK/ERK and PI3K activation, resulting in aggressive, immune-suppressed tumors. Combined inhibition of MEK and p110 $\beta$ /PI3K reduces mouse tumor cell growth in vitro. Therapeutic inhibition of MEK and p110 $\beta$ /PI3K using Selumetinib (AZD6244, ARRY-142886) and AZD8186, two drugs that are currently in clinical trials, increases the survival of Pten;Trp53-null mice without major toxicity. This drug combination effectively reduces the proliferation of primary cultures of human pleural (Pl) $\mathrm{MM}$, implicating non-epithelioid histology and high Vimentin, AKT1/2, and Gai2 expression levels as predictive markers of response to combined MEK and p110ß/PI3K inhibition. Our findings provide the rationale for the use of Selumetinib and AZD8186 in MM patients with sarcomatoid features. This constitutes a novel targeted therapy for a poor prognosis and frequently chemoresistant group of MM patients, for whom therapeutic options are currently lacking.
\end{abstract}




\section{Introduction}

Malignant Mesothelioma (MM) arises mainly from the pleural (pl) and peritoneal (pe) mesothelium and less frequently from other sites and it is strongly associated with asbestos exposure. There are three main histologic subtypes: epithelioid (EMM), accounting for approximately $60 \%$ of MM; sarcomatoid (SMM), constituting approximately 20\%; and biphasic (BMM). BMM contain a variable proportion of tumor cells with epithelioid and sarcomatoid features. Patients with MM have an extremely poor prognosis, especially when the tumor displays sarcomatoid features (pure or biphasic), in part due to its aggressiveness and chemoresistance. Even with multimodality treatment, median survival is 14-18 months (1). There is currently no standard second-line treatment for MM and Bevacizumab is the only approved targeted therapy, in combination with cisplatin and pemetrexed (2). This is due, in part, to the limited understanding of the molecular pathogenesis of MM. Genetic analyses have allowed classifying MM and identifying druggable pathways (3-7). Sarcomatoid MM cases are underrepresented in all reported cohorts, constituting 3-9\% of all specimens, hampering their molecular characterization and the identification of genetic alterations contributing to their poor prognosis and chemoresistance. Recently proposed molecular classifications of $\mathrm{MM}$ agree on the existence of a poor prognosis subtype which includes sarcomatoid tumors. TP53 alterations and PI3K-mTOR and RAS/MAPK upregulation were proposed to associate with the non-epithelioid clusters (6-8).

The PI3K/AKT/mTOR pathway promotes cell proliferation and survival and is activated in most MM (9-11). The mechanisms leading to PI3K/AKT/mTOR activation have not been completely elucidated. Point mutations in genes coding for PI3K pathway components are rare in MM $(6,12)$ but loss of expression of PTEN, the lipid phosphatase that dephosphorylates the main PI3K product [PtdIns(3,4,5)P3] (PIP3), appears much more frequent (16-62\%) measured by immunohistochemical assays (13-16). Genetic MM mouse models, sometimes combined with asbestos exposure, have confirmed the key role of PI3K/mTOR activation and Trp53 inactivation in MM initiation and progression (17-19). 
Indeed, combined Pten and Trp53 deletion in mouse pleural or peritoneal mesothelium leads to sarcomatoid and biphasic MM (19).

PI3K members belong to three classes; class I PI3K are subdivided into IA and IB based on their structure and the activating mechanisms. Class IA PI3K members are heterodimers of a catalytic (p110) and a regulatory subunit (p85). Four genes (PIK3CA, PIK3CB, PIK3CD, and PIK3CG) code for individual p110 isoforms (p110 $\alpha, \mathrm{p} 110 \beta, \mathrm{p} 110 \gamma, \mathrm{p} 110 \delta) \cdot \mathrm{p} 110 \alpha$ and $\mathrm{p} 110 \beta$ are ubiquitous while $\mathrm{p} 110 \delta$ and $\mathrm{p} 110 \gamma$ expression is enriched in hematopoietic cells. p110 $\alpha$ signals downstream of receptor tyrosine kinases (RTKs) while p110 $\beta$ has a prominent role downstream of GPCRs (20,21). PIP3 activates AKT which, in turn, signals to mTOR via TSC1/2 phosphorylation.

Here we report that concurrent PI3K activation and Trp53 deletion in the peritoneal mesothelium leads to aggressive sarcomatoid MM, confirming previous findings (19). We find that Pten deletion drives more aggressive tumors than Pik3ca mutational activation. We confirm that human SMM display low PTEN expression. These mouse model-based strategies allowed identifying the mechanisms responsible for the aggressiveness of SMM, which revealed combined p110 $/$ MEK inhibition as a novel targeted therapy for MM with sarcomatoid features.

\section{Materials and Methods}

Mouse strains. Pten ${ }^{l o x / l o x}$ and $\operatorname{Trp} 53^{l o x / l o x}$ strains were previously reported $(22,23)$. To generate $P i k 3 c a^{H 1047 R}$ knockin mice, the Pik3ca region encompassing exon 18 to 3'UTR, comprising exons 18-20, was substituted by homologous recombination by a cDNA including wild type exons 18-20-3'UTR followed by PGK-NEO for Neomycin selection. These sequences were flanked by LoxP sites; following the 3' LoxP site, the recombinant sequence included the cDNA of exons 18-20 with the $3141 \mathrm{~A}<\mathrm{G}$ substitution, coding for the H1047R mutation, followed by IRES GFP-Luc, and the 3'UTR (Fig. 1A). The construct was electroporated into embryonic stem cells (ESC), which were selected with neomycin. The recombinant sequence was analyzed by PCR. Positive cells were transferred into pseudo-pregnant female 
mice. ESC electroporation, selection and transfer followed standard protocols. Mice born after ESC transfer were genotyped by Southern blot and PCR, crossed with wild-type (WT) C57BL/6 mice, and pups were genotyped to verify germ line transmission. Mice from all the indicated strains (mixed background) were bred and maintained in specific pathogen-free conditions. All animal procedures were approved by the Ethics Committee for Research and Animal Welfare of Instituto de Salud Carlos III and the General Guidance of the Environment of Madrid Community and performed following guidelines for Ethical Conduct in the Care and Use of Animals as stated in The International Guiding Principles for Biomedical Research involving Animals, developed by the Council for International Organizations of Medical Sciences.

Histopathology, immunohistochemistry, and RNAscope. Mouse tissues were dissected, fixed in $10 \%$ buffered formalin, and paraffin-embedded. Human MM tissue microarrays (TMAs) and associated data were obtained through the National Mesothelioma Virtual Bank (University of Maryland, MD; University of Pittsburgh Medical Center, PA; and Roswell Park Cancer Institute, NY, USA). Antigen retrieval was performed by boiling in citrate buffer $\mathrm{pH}$ 6.0; endogenous peroxidase was inactivated with $3 \% \mathrm{H}_{2} \mathrm{O}_{2}$ in methanol. Sections were blocked, rinsed, and incubated with specific antibodies (Supplementary Table S1) and species-specific secondary HRP-conjugated antibodies (EnVision ${ }^{\mathrm{TM}}$ Systems, DAKO-Agilent Technologies). Reactions were developed using DAB (DAKO-Agilent Technologies). Histoscores of human TMAs were calculated as the product of the proportion of reactive cells (0-100\%) and intensity (0-3). The average of the histoscores from each patient was used for analysis. RNAscope in situ hybridization for PD-L1 and Ppib (used for quality control) on formalin-fixed paraffinembedded mouse tumors were performed using the RNAscope 2.5 HD Assay-RED (Advanced Cell Diagnostics) according to manufacturer's instructions. For mouse immunohistochemistry and RNAscope quantification, hematoxylin and antigen-specific signals were detached into separated channels by Colour Deconvolution plugin of Image $\mathbf{J}$ software and channel-specific pixel area quantified using the same 
software. DAB area was normalized to the hematoxylin area; PD-L1 RNAscope signal was normalized to Ppib signal.

Western blotting (WB) and protein-based assays. Frozen tissues and cultured cells were lysed in radioimmunoprecipitation buffer (20mM Tris- $\mathrm{HCl}, \mathrm{pH} 8.0,137 \mathrm{mM} \mathrm{NaCl}, 1 \mathrm{mM} \mathrm{MgCl} 2,1 \mathrm{mM} \mathrm{CaCl}{ }_{2}$, $10 \%$ glycerol, $1 \%$ NP40, $0.5 \%$ sodium deoxycholate, $0.1 \%$ sodium dodecyl sulfate [SDS]) containing protease and phosphatase inhibitors. Proteins fractionated by SDS-PAGE were transferred to nitrocellulose membranes, which were incubated with primary antibodies (Supplementary Table S1) and HPRP-conjugated secondary antibodies (DAKO-Agilent Technologies). Reactions were developed using Luminata Classico HRP substrate (Merck-Millipore). Phospho-RTK array (R\&D Systems); RAS Activation Assay kits (Merck-Millipore) were used following manufacturer's guidelines. Image J software was used for WB signal quantification.

$R N A$ analysis: SNaPshot, and $R T-q P C R$. Total RNA was isolated from frozen tissues and cultured cells using GenElute Mammalian Total RNA Miniprep Kit (Sigma-Aldrich). DNA was eliminated using the DNA-free kit (Ambion-Life Technologies). Reverse transcription was performed using TaqMan® reverse transcription reagents (Roche-Life Technologies); for PCR, an ABIPRISM 7900HT instrument (Applied Biosystems-Life Technologies) and the SYBR Green PCR Master Mix (Applied BiosystemsLife Technologies) were used. Changes in gene expression were calculated using the quantitative $\Delta \Delta \mathrm{Ct}$ method and normalized against Hprt. To assay for expression of mutant Pik3ca mRNA, a region of the gene encompassing exons 19 and 20 was amplified by PCR from cDNA. PCR products were treated with ExoSAP-IT (Affymetrix-Thermo Fisher Scientific) and the wt and the H1047R alleles were identified using a specific primer and the ABI PRISM SNaPshot Multiplex Kit (Applied Biosystems-Life Technologies). All primer sequences are provided in Supplementary Table S2.

RNA-sequencing. Total RNA $(2 \mu \mathrm{g})$ from Pik3ca*;Trp53-null and Pten;Trp53-null mice (n=6 each) was used. RNA Integrity Numbers ranged from 7.5-9.4, assayed on an Agilent 2100 Bioanalyzer. PolyA+ RNA was extracted and randomly fragmented, converted to double stranded cDNA, and 
processed through enzymatic treatments of end-repair, dA-tailing, and adapter ligation following "TruSeq RNA Sample Preparation Guide". The library was produced by limited-cycle PCR with Illumina PE primers ( 8 cycles). The purified cDNA library was applied to an Illumina flow cell for cluster generation (TruSeq cluster generation kit v5) and sequenced on the Genome Analyzer IIx with SBS TruSeq v5 reagents.

RNA-seq data processing and analyses. Image analysis and per-cycle base-calling was performed with Illumina Real Time Analysis software (RTA1.13). Conversion to FASTQ read format with the ELAND algorithm (v2e) was performed with CASAVA-1.8 (IlluminaQuality check was done via fastqc v0.9.4, Babraham Bioinformatics). Raw reads were aligned with Tophat5 (version 2.0.4) to mouse genome GRCm38/mm10. Gene expression normalized counts (TPMs) and differential expression (Pik3ca*;Trp53-null vs. Pten;Trp53-null mice) was done with DeSeq2 (version 2.0.2).

MM transcriptome data. RNAseq data (6) were downloaded from the European Genomephenome Archive under accession code EGAS00001001563; data were normalized by the Upper Quartile method. Normalized data from the TCGA were downloaded from cBioPortal for Cancer Genomics (24).

Cell culture. For mouse tumor cell isolation, tumors were digested in NB8 collagenase (1.5 $\mathrm{mg} / \mathrm{mL}$ in HBSS) (LabClinics) and maintained in DMEM supplemented with $10 \% \mathrm{FBS}, 1 \mathrm{mM} \mathrm{Na}$ pyruvate and non-essential amino acids (Gibco-Life Technologies), and incubated at $37{ }^{\circ} \mathrm{C}$ in a $5 \% \mathrm{CO}_{2}$ and $3 \% \mathrm{O}_{2}$ atmosphere. All experiments were performed at $<15$ passage. Human primary mesothelioma cultures (MPM.04, 12, 24, 28, 29, 34, 35, 37, 47, 59 and 60) were established at INSERM U.1138. MESO.49, 62, 80, 84, 92, 103, 161 and VMC.40, 48 were established at the Medical University of Vienna. Cells were grown either in RPMI 1640 (VMC40 and Meso161) or in DMEM supplemented with Glutamax (Gibco-Life Technologies) and $10 \% \mathrm{FBS}$ at $37^{\circ} \mathrm{C}$ in a $5 \% \mathrm{CO}_{2}$ atmosphere.

Reagents and antibodies. PD0325901 MEK inhibitor (1408) was purchased from AxonMedchem, TGX-221 (S1169) was obtained from Selleckchem, Pertussis toxin (P7208) and Cisplatin 
(C2210000) were from Sigma-Aldrich (P7208). Selumetinib and AZD8186 were kindly provided by AstraZeneca.

In vitro invasion and proliferation assays. In vitro invasion assays were performed using BioCoat $^{\mathrm{TM}}$ Matrigel $^{\mathrm{TM}}$ Invasion Chambers (Corning) following manufacturer's guidelines. Cells were fixed with $4 \%$ PFA and nuclei were stained with DAPI $(0.5 \mu \mathrm{g} / \mathrm{mL}$ in PBS). Cells were visualized using a Leica TCS SP5 WLL confocal microscope. IMARIS software v5.0 (Bitplane) was used for 3D image reconstruction and nuclear quantification. Mouse cell proliferation assays were performed in 96-well plates (Greiner Bio-one). 24h after plating at $5 \times 10^{3}$ cells/well (triplicates), inhibitors or vehicle were added; cells were incubated until fixation with 4\% PFA. Visualization and image acquisition of DAPI stained nuclei were performed using the confocal microscopy-based PerkinElmer's Opera ${ }^{\circledR}$ high content screening system. Nuclei were counted using Acapella software (PerkinElmer). For proliferation assays, human primary cells were seeded at $5-7 \times 10^{3}$ cells/well in triplicate in 96-well plates (Corning, Falcon). Cells were treated for $72 \mathrm{~h}$ with increasing concentrations of single, or both, drugs; viability was quantified by CellTiter 96 AQueous One Solution Cell Proliferation Assay (MTS) (G3582) or CellTiter Glo Luminescent Cell Viability Assay (G7573) (Promega). The combination ratios for Selumetinib and AZ8186 are specified in Supplementary Table S3. Area Under Curve (AUC) and GI50s for single drugs were calculated using GraphPad software 5.0 (Prism). Combination drug GI50 and CI were determined by Compusyn software (http://www.combosyn.com/).

Lentiviral production and Gai2 knockdown. Gai2 was knocked-down in mouse mesothelioma cells using MISSION shRNA Gai2-shRNAs (SHC002, SHCLNG-NM_008138, and control sh; Sigma Aldrich). Targeting sequences are provided in Supplementary Table S2. Lentiviruses were produced in $293 \mathrm{~T}$ cells using calcium phosphate transfection. Viral supernatant was collected $48 \mathrm{~h}$ and $72 \mathrm{~h}$ after transfection. Lentiviruses were transduced into mouse mesothelioma cells $(0.2 \times 106$ cells $/ 6$ well-plate $)$ followed by Puromycin selection $(1 \mu \mathrm{g} / \mathrm{ml}, 48 \mathrm{~h})$. Cells were harvested 24 hours later. 
In vivo experiments. CT tumor-bearing Pten;Trp53-null mice were randomized into control and treatment groups. Selumetinib $(10 \mathrm{mg} / \mathrm{kg})$ and AZD8186 $(40 \mathrm{mg} / \mathrm{kg})$ were administered by oral gavage, twice daily, 5 days/week. Control mice received vehicle ( $0.5 \%$ methylcellulose, $0,2 \%$ Tween 80$)$.

MicroCT imaging. Mice were anesthetized and the abdominopelvic area was imaged using the eXplore Vista micro-CT scanner (GE Healthcare) without contrast. Micro-CT image acquisition consisted of 400 projections collected in one full rotation of the gantry in approximately 10 min using $80 \mathrm{kV}$ and $450 \mu \mathrm{A}$ X-ray tube settings. Reconstructed images were viewed and analyzed using AMIDE software.

Statistical analyses. Mann-Whitney U-Test was used to compare differences between two independent groups when the data did not follow a normal distribution. Kruskal-Wallis one-way analysis of variance was used when more than two groups were compared. Log-rank (Mantel-Cox) test was used to compare survival distributions. Fisher's exact test was used in the analysis of the distribution of categorical values within two groups. GraphPad software 5.0 (Prism) was used. Human data statistics were performed by R software, ANOVA test was used to compare differences between group means, and Tukey Test was applied as post-hoc test. Two-sided $\mathrm{P}$ values $<0.05$ were considered significant.

\section{Results}

Trp53 loss cooperates with both Pik3ca mutation and Pten loss in mouse MM. Peritoneal MM developed in 8-12 week-old $\operatorname{Pten}^{\text {lox/lox}} ; \operatorname{Trp} 53^{\text {lox/lox }}$ mice (referred to herein as Pten;Trp53-null) following inoculation of adenoviruses coding for Cre-recombinase into the bladder wall, as described (Fig. 1B, Supplementary Information) (18). Recombination was evaluated using R26-LSL-LacZ reporter mice two weeks later (Fig. 1C, Supplementary Information). All inoculated Pten;Trp53-null mice (n=26), but only $2 / 11 \operatorname{Trp} 53^{l o x / l o x}$ mice, developed tumors in the peritoneal aspect of the bladder wall, frequently invading contiguous fat and muscle (Fig. 1D). In addition, 15/26 (57\%) mice developed peritoneal lesions. Tumor histology and latency recapitulate previously described Trp53;Pten-null non-epithelioid MM (19). Tumors were pleomorphic, enriched in spindle-cells, and showed mild infiltration by lymphocytes and granulocytes. Immunohistochemistry showed heterogeneous but consistent expression of WT1 (9/9) and 
vimentin (12/12) and diffuse KRT5 expression in 4/12 tumors (Fig. 1E). These features, together with the histology, are characteristic of SMM.

Given the relevance of the PI3K/AKT/mTOR pathway in MM cell proliferation and survival $(9,10,18)$ we assessed more broadly the impact of PI3K activation in a Trp53-null context. We analyzed $P i k 3 \mathrm{ca}^{\text {wt/H1047R }}$;Trp53 $3^{l o x / l o x}$ mice (referred to as Pik3ca*;Trp53-null), in which the H1047R hotspot mutation was conditionally knocked in the Pik3ca locus (Fig. 1A). After adeno-Cre inoculation, all Pik3ca*;Trp53null mice developed tumors histologically undistinguishable from those of Pten;Trp53-null mice (Fig. 1E). However, the median survival of Pik3ca*;Trp53-null mice was significantly longer than that of Pten;Trp53-null mice (17.6 vs. 10.9 weeks after adenoviral inoculation, $P<0.0001$ ) (Fig. 1F). Importantly,

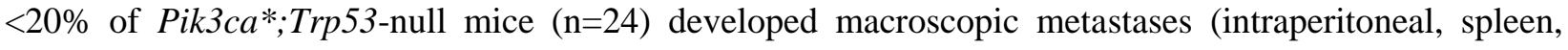
kidneys, liver and gastrointestinal tract), compared with 50\% of Pten; Trp53-null mice $(\mathrm{n}=14)(P=0.024$, Fisher's exact test) (Supplementary Fig.S1).

Immunohistochemical analysis revealed that Pten;Trp53-null tumors displayed higher proliferation (phospho-Histone 3) and increased inflammation (macrophages and $\mathrm{T}$ cells) than Pik3ca*;Trp53-null tumors; with no differences in apoptosis ( cleaved-Caspase 3) (Fig. 2). A refined analysis revealed increased $\mathrm{Cd} 8^{+}$cells, $\mathrm{Ym}^{+} \mathrm{M} 2$ macrophages, and $\mathrm{Cd} 4^{+} \mathrm{FoxP}^{+} \mathrm{T}$ regs in Pten;Trp53null tumors (Fig. 2C and D). RNAscope analysis showed discrete areas of PD-L1-expressing cells in Pten;Trp53-null tumors, but not in Pik3ca*;Trp53-null lesions (Fig. 2E and F). These data indicate that Pten inactivation associates with a more immunosuppressive stroma and metastatic phenotype than Pik3ca activation.

Human SMM display low PTEN expression. Given the relevance of Trp53 inactivation and PI3K activation in mouse SMM, we interrogated the PI3K pathway and upstream regulators in human SMM. PTEN loss, assesed by immunohistochemistry, has been reported in 12-62\% of unselected MM (11,13-15) but its relevance to the sarcomatoid phenotype is unknown. PTEN expression was analyzed in TMAs including 149 clinically annotated specimens (National Mesothelioma Virtual Bank, Supplementary Table 
S4) (25). PTEN immunostaining scores were categorized as negative (0), weak (1), moderate (2), and strong (3) (Fig. 3A): $15.4 \%$ tumors scored negative ( $<20$ immunoscore). PTEN-negative cases were enriched in SMM ( $P=0.017$, Fisher's exact test) (Fig. 3B). PTEN immunoscores were significantly lower in SMM than in epithelioid and biphasic tumors (Fig. 3C). Given the heterogeneity of biphasic MMs, we subdivided them according to the sarcomatoid or epitheliod components. Biphasic MMs with <50\% epithelioid cells displayed lower PTEN levels but differences were not significant when compared with those with $>50 \%$ cells $(P=0.134)$. At the transcriptomic level, expression of PTEN was significantly lower in the sarcomatoid group of Bueno (6), while expression of other PI3K effectors was increased. Despite following the same trend, differences were not significant when considering histology groups (Fig. 3D). A similar tendency was observed in the TCGA cohort (7) when clustered similarly (6) (Fig 3E). These data demonstrate that PTEN down-regulation is common in SMM and validate the Pten;Trp53-null mouse model as a valuable tool.

\section{MEK/ERK activation contributes to the growth and invasive capacity of Pten;Trp53-null tumor}

cells. To identify the mechanisms contributing to the aggressiveness of Pten;Trp53-null tumors, we analyzed PI3K and MAPK/ERK activation, known to cooperate in tumor progression. Akt Ser473 and Thr308 phosphorylation was significantly higher in Pten;Trp53-null tumors compared with Pik3ca*;Trp53-null tumors. Remarkably, pErk1/2 (Thr202 and Tyr204) and pMek1/2 (Ser217/221) were also higher in Pten;Trp53-null tumors (Fig. 4A-C). We used RAS-GTP pull-down assays to assess upstream signaling and did not find strong evidence of RAS hyperactivation in Pten;Trp53-null tumors, compared with Pik3ca*;Trp53-null tumors (Supplementary Fig. S2A and S2B). Downstream, we did not find consistent differences in phosphorylation of GSK3 $\beta$ Ser9 nor in the mTOR targets S6K and 4Ebp1 in Pten;Trp53-null tumors (Supplementary Fig. S2C and S2D).

To study the mechanisms involved in MEK/ERK activation and their contribution to the aggressiveness of Pten;Trp53-null tumors, we established murine MM cell lines (Fig. 4D). Expression of mutant Pik3ca mRNA in Pik3ca*;Trp53-null cells was confirmed using a SNaPshot assay 
(Supplementary Fig. S3A). Trp53 and Pten deletion were also confirmed (Fig. 4E and Supplementary Fig. S3B). We found higher pAkt levels in Pten;Trp53-null cells than in Pik3ca*;Trp53-null cells (Fig. 4E and Supplementary Fig. S3C) but the differences in pERK levels in exponentially growing cells from both genotypes were less apparent than in tumor tissues. MEK1/2 inhibition with PD325901 led to decreased pErk in cells of both genotypes (Supplementary Fig. S4A). MEK1/2 inhibition led to reduced in vitro proliferation and invasiveness of Pten;Trp53-null cells whereas only proliferation was affected in Pik3ca*;Trp53-null cells (Fig. 4F and 4G). These results indicate that MAPK pathway activation contributes to the aggressiveness of Pten;Trp53-null tumors, although additional signaling pathways must be implicated.

GPCRs mediate Erk and PI3K activation in Pten;Trp53-null tumor cells. MAPK activation resulting from PTEN loss has been reported but no underlying unifying mechanism has been identified (26). A role of RTK - such as EGFR, EpHB4, MET and AXL - in MAPK activation has been proposed in MM (10,27). To explore if such mechanisms are involved in the differential activation of MAPK in Trp53;Pten-null mice, tumor lysates were analysed by p-RTK dot blot arrays. We did not find strong evidence for a role of hyperphosphorylation of any of the 39 RTK tested. By contrast, selected receptors were preferentially hyperphosphorylated in Pik3ca*;Trp53-null tumors (Fig. 5A and 5B). Altogether, these results do not support RTK/RAS as the predominant mechanism responsible for MAPK activation in aggressive Pten;Trp53-null MM.

Next, we assessed whether GPCRs may participate in MAPK activation. To explore this notion, we used Pertussis toxin (PTX) which induces ADP-ribosylation of the $\alpha$ i subunits of heterotrimeric G proteins and impaired interaction with GPCRs, thus preventing downstream pathway activation (28). Erk phosphorylation was markedly reduced in PTX-treated Pten;Trp53-null cells but not in Pik3ca*;Trp53null cells while Akt phosphorylation was only modestly affected (Fig. 5C). The Gai family consists of Gai1-3, Gao, Gat1-3, and Gaz. RNA-Seq data showed that Gai2 is the main Gai subunit expressed in mouse SMM, regardless of the host genotype (Fig. 5D). Gai2 expression was confirmed at the protein 
level in all tested Pten;Trp53-null (n=8) and Pik3ca*;Trp53-null (n=9) MM by immunohistochemistry, with a heterogeneous expression pattern (Fig. 5E). To confirm the role of GPCRs signaling in MAPK activation in Pten;Trp53-null cells, we targeted Gai2 using shRNAs. Gai2 knockdown efficiently and consistently reduced Erk and Akt phosphorylation in Pten;Trp53-null, but not in Pik3ca*;Trp53-null cells (Fig. 5F). These results provide novel evidence on the involvement of GPCRs in MAPK and PI3K activation in Pten;Trp53-null MM cells. To further assess the relevance of this pathway in human MMs, we interrogated expression in the series from Bueno et al. (6), finding that GNAI2 expression is significantly up-regulated in sarcomatoid transcriptome- and histology-defined subgroups (Fig. 5G).

\section{P110 $\beta$ and Mek1/2 inhibitors cooperate to suppress mouse Pten;Trp53-null tumors growth.}

p110 $\beta$ has been shown to play a crucial role in PTEN-null tumors in vitro and in vivo $(29,30)$ and is mainly activated by GPCRs (21). We hypothesized that $\mathrm{p} 110 \beta$ and Mek1/2 cooperate driving the aggressive phenotype of Pten;Trp53-null tumors. TGX-221, a selective p110 $\beta$ inhibitor, reduced PI3Kdependent Akt phosphorylation (Thr308) to a greater extent in Pten;Trp53-null cells than in Pik3ca*;Trp53-null cells. Surprisingly, mTORC2-dependent phosphorylation of Akt (Ser473) was inhibited in all cell lines (Supplementary Fig. S4B). TGX-221 had no effect on pErk, suggesting that combined inhibition of $\mathrm{p} 110 \beta$ and MEK1/2 might be more active in Pten;Trp53 cells.

As single agents, TGX-221 and PD325901 had modest anti-proliferative effect. However, their combination completely suppressed the proliferation of Pten;Trp53-null cells. Both inhibitors decreased in vitro invasiveness (Fig. 6A and 6B). Based on the in vitro efficacy of simultaneous MEK and p110 $\beta$ inhibition, we tested the combination in vivo using drugs undergoing clinical investigation. Selumetinib is a selective MEK1/2 inhibitor in phase III trials for NSCLC, thyroid cancer, and metastatic uveal

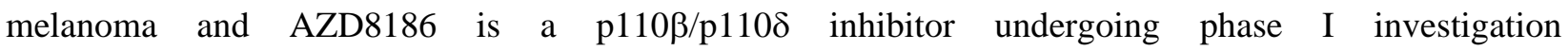
(https://clinicaltrials.gov). Pten;Trp53-null tumor growth was followed by weekly micro-CT; early tumor detection was facilitated by bone differentiation, a common feature of MM (Fig. 6C). Once tumors were detected (6-7 weeks after Cre inoculation), mice ( $n=6 /$ group) were randomized to receive daily oral 
treatment with vehicle, Selumetinib, AZD8186, or Selumetinib/AZD8186 for 7 weeks. Single compound treatment did not affect survival. In contrast, a significant lifespan increase $(P=0.031)$ was observed with the combination (Fig. 6D). Accordingly, CT images showed increased size and number of lesions in vehicle- and single compound-treated mice, and reduced tumor progression upon drug combination (Fig. $6 \mathrm{E})$. The dependency of tumor shrinkage on treatment was shown upon its discontinuation: within the following 5 weeks, tumors grew in 5 mice but one surviving mouse sacrificed 26 weeks later showed a complete pathological response (Supplementary Fig. S5A and S5C). In an independent experiment, CT tumor-bearing Pten;Trp53-null mice were randomized to receive vehicle or Selumetinib/AZD8186 $(\mathrm{n}=11)$. Treatment was maintained for 12 weeks without toxicity. Median survival of treated Pten;Trp53null mice was 13.4 weeks, compared with 9.3 weeks for control mice $(P=0.005)$ (Fig. 6F). Of two surviving mice, one had a minimal peritoneal tumor and the other displayed only microscopic tumor foci at autopsy (Supplementary Fig. S5B, S5D and S5E).

P110 $\beta$ and Mek1/2 inhibitors cooperate to suppress human pl-MM cell growth. We next analyzed the effects of this drug combination on 20 human primary pl-MM cultures [EMM $(n=8), B M M(n=7)$ and SMM (n=5)] (4,31-33). Unfortunately, no primary pe-MM were available for these studies. Response was defined as a $>50 \%$ reduction of cell viability and combination GI50 $<10 \mu \mathrm{M}$ (calculated as the sum of GI50s of each drug). Six of $12 \mathrm{BMM} / \mathrm{SMM}$ and 1/8 EMM cultures responded to the combination (Fig. 7A, 7B, and Supplementary Fig. S6A); in 6 of the responding cultures Selumetinib and AZD8186 were synergistic, displaying a combination index (CI) $<1$ (Supplementary Table S5). BMM and SMM also displayed lower GI50s to Selumetinib/AZD8186 combination (Supplementary Fig. S6B and S6C). AUC responses to increasing drug concentrations revealed that BMM and SMM were more sensitive to AZD8186 $(P=0.015)$, and to the combination $(P=0.049)$, than EMM (Fig. 7B and 7C). To further define predictive markers of response to the Selumetinib/AZD8186 combination, we assessed Vimentin, Gai2, TP53, and MAPK and PI3K pathways status in lysates from primary human MM cells (Fig. 7D) and found that low Vimentin, Gai2, and AKT levels identify the subgroup of resistant cells (Fig 7E). Finally, 
to explore the potential clinical relevance of these findings, we compared the effects of Selumetinib/AZD8186 combination to cisplatin (Supplementary Fig. S7): responder cells displayed higher sensitivity to Selumetinib/AZD8186 than to cisplatin $(P=0.038)$ (Fig. 7F). Altogether these results point to Selumetinib/AZD8186 as a novel therapy alternative to Cisplatin for pl- an pe- non-epithelioid MM patients, high expression of Vimentin, AKT, and Gai2 being candidate biomarkers predictive of response.

\section{Discussion}

There is an urgent need to understand the molecular factors linked to sarcomatoid MM subtype and their aggressiveness to define therapeutic improvements. EMT may, in part, explain the poor prognosis of SMM, conferring both high invasiveness and chemoresistance $(4,6,7)$. Recent reports highlight genetic alterations and molecular mechanisms associated to EMT in SMM, such as TP53 inactivation, LATS2 mutations, CDKN2A homozygous deletions, E2F target up-regulation, lncRNA expression, and PI3K-mTOR and RAS/MAPK activation (4,6,7,34). Here, we confirmed the relevance of concomitant Trp53 loss and PI3K activation as drivers of mouse SMM. A few genetic models of MM have previously been reported based on the inactivation of Nf2, Bapl, Ink4a/Arf, Trp53, Tsc1 and Pten with or without the combined administration of asbestos. In most cases, the 3 major histological subtypes of MM developed but only $N f 2 ; R b$ and Pten;Trp53 combined genetic loss leads to exclusively nonepithelioid $(17,19,35)$. In our hands, adenovirus-Cre mediated Pten;Trp53 inactivation resulted in SMM development with a median latency and histology similar to those reported by Sementino et al. but we did not observe biphasic MM, as they did. These differences might reside in the genetic background used.

PI3K/AKT/mTORC1 and ERK pathway activation occurs in most human MM specimens, thought to be a consequence of RTK activation $(9,10,18)$, although anti-RTKs therapies have shown limited effectiveness $(16,36)$. The role of PTEN, and its implication in PI3K pathway activation in MM, is controversial as few genetic alterations have been identified in this gene. However, PTEN can be inactivated through multiple mechanisms (37). Thus, PTEN loss has been reported in $16-62 \%$ of cases

using immunohistochemistry $(11,13-15)$ and a recent case report associates PTEN-hamartoma-tumor- 
syndrome with MM development pointing to PTEN loss as a MM driver (38). Most studies relating PTEN and MM did not discriminate between histologic MM subtypes. We show using human MM TMAs that PTEN protein loss is frequent in SMM and that this subtype displays lower PTEN expression, compared with epithelioid and biphasic MM. The analysis is limited by the low number of sarcomatoid samples in our cohort $(\mathrm{n}=7)$ but PTEN mRNA levels were lower in transcriptomic defined sarcomatoid tumors when compared with the other MMs. PTEN expression in biphasic MMs is paradoxal, with higher histoscores in sarcomatoid tumors. Biphasic MM constitutes a highly heterogeneous group with variable proportions of epithelioid and sarcomatoid cells. These differences were highlighted in the transcriptomic Biphasic-E and Biphasic-S clusters defined by Bueno et al. (6). In addition, the relevance of intratumor heterogeneity regarding epithelioid and sarcomatoid components was recently underscored by Blum et al. (8), indicating that a more precise definition of the biphasic group is required. The reported low PTEN levels in sarcomatoid MMs, plus the reported association of TP53 mutations with non-epithelioid MM, underline the relevance of Trp53;Pten-null mice as a MM pre-clinical model $(6,8,19)$.

Our data also revealed that Pten loss has stronger oncogenic properties than Pik3ca mutations, favoring metastases and an immunosuppressive environment enriched in Cd8 $\mathrm{T}$ cells, $\mathrm{T}$ regs, M2 macrophages, and PD-L1-expressing cells that have been associated with poor prognosis in human MM (39-41). Remarkably, non-epithelioid human MMs exhibit higher PD-L1 expression and T cell infiltration $(6-8,41)$. These observations are in agreement with the fact that PTEN antagonizes further PI3K isoforms implicated in the activation of pathways other than mTOR, and that it exerts lipid phosphataseindependent functions (42). Biochemical analysis of Pik3ca*;Trp53-null and Trp53;Pten-null tumors revealed strong Akt and Mek/Erk activation without major evidence for a role of upstream RTKs. This allowed us to uncover a novel role of GPCR/Gai2 signaling in Erk phosphorylation specifically in Trp53;Pten-null, but not in Pik3ca*;Trp53-null, cells. Importantly, Gai2 mRNA levels are upregulated in human sarcomatoid MMs when compared with the other subgroups and its signaling has been implicated in the proliferation and migration of ovarian and prostate cancer cells $(43,44)$. 
Several observations led us to focus on $110 \beta$ as a crucial mediator of the Trp53;Pten-null tumor phenotype. p1 $10 \beta$ has been shown to essential for tumorigenesis in a PTEN-null context $(29,30)$. In addition, p110 $\beta$ can be activated by both RTKs and GPCRs but genetic data suggest a minor impact of p110 $\beta$ deletion, or its lipid kinase inactivation, on RTK signaling (21). In vitro, only a fraction of PTENnull cancer cells are sensitive to p110 $\beta$ inhibitors and in vivo studies have not consistently shown tumor regression (45,46). As single-agent, the dual PI3K and mTOR inhibitor Apitolisib (GDC0980) showed modest antitumor activity in a phase I trial that included patients with advanced MM (47), indicating that PI3K inhibitors need to be combined with other drugs to achieve greater antitumor effects. The MAPK pathway activation observed in Trp53;Pten-null tumors pointed to the combination of $\mathrm{p} 110 \beta$ and MEK inhibitors. Indeed, we observed that p110ß (TGX221) and MEK (PD325901) inhibitors show synergy on the growth of Trp53;Pten-null tumor cells. More importantly, the combination of two drugs that are currently under clinical investigation and target MEK and $\mathrm{p} 110 \beta$ revealed strong antitumor effect in vivo in established Trp53;Pten-null tumors, including induction of complete pathological responses. Several early-phase clinical studies using MEK and PI3K inhibitors are ongoing with significant toxicity (48), but we did not encounter toxicity in mice. Recent data point that the use of isoform-selective PI3K inhibitors would allow optimizing combined therapy and reducing toxicity (49). Accordingly, p110ß/MEK inhibition has been proposed in preclinical settings for PTEN-deficient/BRAF-mutated melanoma (50).

One major reason for the inability to reproduce promising results of preclinical studies in clinical trials is the lack of adequate patient stratification. Here, we provide evidence pointing to the nonepithelioid histology and high Vimentin, AKT1/2, and Gai2 protein levels as predictive markers of response to Selumetinib/AZD8186 therapy by testing this combination on 20 primary cultures derived from epithelioid, biphasic, and sarcomatoid human pl-MM. Responder cells presented significantly lower GI50s to combined therapy than cisplatin. We propose that this combination be tested in patients with advanced MM with biphasic/sarcomatoid features, a subgroup for which therapeutic options - including 
surgery - are scarce. Should the acceptable toxicity observed in mice hold in patients, the combination of Selumetinib/AZD8186 with cisplatin would also merit consideration.

In summary, we identify a novel druggable molecular pathway driven by GPCRs/MEK/p110 $\beta$ signaling, which has a major impact on MM progression using human-relevant genetic mouse models and represents a novel targeted therapy for patients with MM whose tumors display sarcomatoid features. 


\section{Acknowledgements}

We thank E. Andrada, N. Malats, M. Márquez, M. Rava, V. J. Sánchez-Arévalo Lobo, and F. Larousserie for valuable contributions; the Imaging and Histopathology CNIO Units for help with CT and histology interpretation; and M. Barbacid, R. García, X. Bustelo, A. C. Carrera, P. Smith, and A. Nebreda for valuable comments to earlier versions of the manuscript. AZ provided drug (as required by AZ publication policy).

\section{Data and material availability}

Raw and processed sequencing data are available in The Gene Expression Omnibus (GEO) repository (GSE138389). 


\section{BIBLIOGRAPHY}

1. Yap TA, Aerts JG, Popat S, Fennell DA. Novel insights into mesothelioma biology and implications for therapy. Nat Rev Cancer 2017;17:475-88

2. Zalcman G, Mazieres J, Margery J, Greillier L, Audigier-Valette C, Moro-Sibilot D, et al. Bevacizumab for newly diagnosed pleural mesothelioma in the Mesothelioma Avastin Cisplatin Pemetrexed Study (MAPS): a randomised, controlled, open-label, phase 3 trial. Lancet 2016;387:1405-14

3. Christensen BC, Houseman EA, Poage GM, Godleski JJ, Bueno R, Sugarbaker DJ, et al. Integrated profiling reveals a global correlation between epigenetic and genetic alterations in mesothelioma. Cancer Res 2010;70:5686-94

4. de Reynies A, Jaurand MC, Renier A, Couchy G, Hysi I, Elarouci N, et al. Molecular classification of malignant pleural mesothelioma: identification of a poor prognosis subgroup linked to the epithelial-to-mesenchymal transition. Clin Cancer Res 2014;20:1323-34

5. Yoshikawa Y, Emi M, Hashimoto-Tamaoki T, Ohmuraya M, Sato A, Tsujimura T, et al. Highdensity array-CGH with targeted NGS unmask multiple noncontiguous minute deletions on chromosome 3p21 in mesothelioma. Proc Natl Acad Sci U S A 2016;113:13432-7

6. Bueno R, Stawiski EW, Goldstein LD, Durinck S, De Rienzo A, Modrusan Z, et al. Comprehensive genomic analysis of malignant pleural mesothelioma identifies recurrent mutations, gene fusions and splicing alterations. Nat Genet 2016;48:407-16

7. Hmeljak J, Sanchez-Vega F, Hoadley KA, Shih J, Stewart C, Heiman DI, et al. Integrative Molecular Characterization of Malignant Pleural Mesothelioma. Cancer Discov 2018

8. Blum Y, Meiller C, Quetel L, Elarouci N, Ayadi M, Tashtanbaeva D, et al. Dissecting heterogeneity in malignant pleural mesothelioma through histo-molecular gradients for clinical applications. Nat Commun 2019;10:1333

9. Altomare DA, You H, Xiao GH, Ramos-Nino ME, Skele KL, De Rienzo A, et al. Human and mouse mesotheliomas exhibit elevated AKT/PKB activity, which can be targeted pharmacologically to inhibit tumor cell growth. Oncogene 2005;24:6080-9

10. Zhou S, Liu L, Li H, Eilers G, Kuang Y, Shi S, et al. Multipoint targeting of the PI3K/mTOR pathway in mesothelioma. Br J Cancer 2014;110:2479-88

11. Kanteti R, Dhanasingh I, Kawada I, Lennon FE, Arif Q, Bueno R, et al. MET and PI3K/mTOR as a potential combinatorial therapeutic target in malignant pleural mesothelioma. PLoS One 2014;9:e105919

12. Shukuya T, Serizawa M, Watanabe M, Akamatsu H, Abe M, Imai H, et al. Identification of actionable mutations in malignant pleural mesothelioma. Lung Cancer 2014;86:35-40

13. Opitz I, Soltermann A, Abaecherli M, Hinterberger M, Probst-Hensch N, Stahel R, et al. PTEN expression is a strong predictor of survival in mesothelioma patients. Eur J Cardiothorac Surg 2008; $33: 502-6$

14. Agarwal V, Campbell A, Beaumont KL, Cawkwell L, Lind MJ. PTEN protein expression in malignant pleural mesothelioma. Tumour Biol 2013;34:847-51

15. Cedres S, Ponce-Aix S, Pardo-Aranda N, Navarro-Mendivil A, Martinez-Marti A, Zugazagoitia J, et al. Analysis of expression of PTEN/PI3K pathway and programmed cell death ligand 1 (PDL1) in malignant pleural mesothelioma (MPM). Lung Cancer 2016;96:1-6 
16. Garland LL, Rankin C, Gandara DR, Rivkin SE, Scott KM, Nagle RB, et al. Phase II study of erlotinib in patients with malignant pleural mesothelioma: a Southwest Oncology Group Study. J Clin Oncol 2007;25:2406-13

17. Jongsma J, van Montfort E, Vooijs M, Zevenhoven J, Krimpenfort P, van der Valk M, et al. A conditional mouse model for malignant mesothelioma. Cancer Cell 2008;13:261-71

18. Guo Y, Chirieac LR, Bueno R, Pass H, Wu W, Malinowska IA, et al. Tsc1-Tp53 loss induces mesothelioma in mice, and evidence for this mechanism in human mesothelioma. Oncogene 2014;33:3151-60

19. Sementino E, Menges CW, Kadariya Y, Peri S, Xu J, Liu Z, et al. Inactivation of Tp53 and Pten drives rapid development of pleural and peritoneal malignant mesotheliomas. J Cell Physiol 2018

20. Fruman DA, Chiu H, Hopkins BD, Bagrodia S, Cantley LC, Abraham RT. The PI3K Pathway in Human Disease. Cell 2017;170:605-35

21. Guillermet-Guibert J, Bjorklof K, Salpekar A, Gonella C, Ramadani F, Bilancio A, et al. The p110beta isoform of phosphoinositide 3-kinase signals downstream of $\mathrm{G}$ protein-coupled receptors and is functionally redundant with p110gamma. Proc Natl Acad Sci U S A 2008; $105: 8292-7$

22. Suzuki A, Yamaguchi MT, Ohteki T, Sasaki T, Kaisho T, Kimura Y, et al. T cell-specific loss of Pten leads to defects in central and peripheral tolerance. Immunity 2001;14:523-34

23. Jonkers J, Meuwissen R, van der Gulden H, Peterse H, van der Valk M, Berns A. Synergistic tumor suppressor activity of BRCA2 and p53 in a conditional mouse model for breast cancer. Nat Genet 2001;29:418-25

24. Gao J, Aksoy BA, Dogrusoz U, Dresdner G, Gross B, Sumer SO, et al. Integrative analysis of complex cancer genomics and clinical profiles using the cBioPortal. Sci Signal 2013;6:pl1

25. Amin W, Parwani AV, Melamed J, Flores R, Pennathur A, Valdivieso F, et al. National Mesothelioma Virtual Bank: A Platform for Collaborative Research and Mesothelioma Biobanking Resource to Support Translational Research. Lung Cancer Int 2013;2013:765748

26. Chetram MA, Hinton CV. PTEN regulation of ERK1/2 signaling in cancer. J Recept Signal Transduct Res 2012;32:190-5

27. Ou WB, Hubert C, Corson JM, Bueno R, Flynn DL, Sugarbaker DJ, et al. Targeted inhibition of multiple receptor tyrosine kinases in mesothelioma. Neoplasia 2011;13:12-22

28. Burns DL. Subunit structure and enzymic activity of pertussis toxin. Microbiol Sci 1988;5:285-7

29. Wee S, Wiederschain D, Maira SM, Loo A, Miller C, deBeaumont R, et al. PTEN-deficient cancers depend on PIK3CB. Proc Natl Acad Sci U S A 2008;105:13057-62

30. Jia S, Liu Z, Zhang S, Liu P, Zhang L, Lee SH, et al. Essential roles of PI(3)K-p110beta in cell growth, metabolism and tumorigenesis. Nature 2008;454:776-9

31. Tranchant R, Quetel L, Tallet A, Meiller C, Renier A, de Koning L, et al. Co-occurring mutations of tumor suppressor genes, LATS2 and NF2, in malignant pleural mesothelioma. Clin Cancer Res 2016

32. Schelch K, Hoda MA, Klikovits T, Munzker J, Ghanim B, Wagner C, et al. Fibroblast growth factor receptor inhibition is active against mesothelioma and synergizes with radio- and chemotherapy. Am J Respir Crit Care Med 2014;190:763-72 
33. Hoda MA, Pirker C, Dong Y, Schelch K, Heffeter P, Kryeziu K, et al. Trabectedin Is Active against Malignant Pleural Mesothelioma Cell and Xenograft Models and Synergizes with Chemotherapy and Bcl-2 Inhibition In Vitro. Mol Cancer Ther 2016;15:2357-69

34. Singh AS, Heery R, Gray SG. In Silico and In Vitro Analyses of LncRNAs as Potential Regulators in the Transition from the Epithelioid to Sarcomatoid Histotype of Malignant Pleural Mesothelioma (MPM). Int J Mol Sci 2018;19

35. Jean D, Jaurand MC. Mesotheliomas in Genetically Engineered Mice Unravel Mechanism of Mesothelial Carcinogenesis. Int J Mol Sci 2018; 19

36. Laurie SA, Gupta A, Chu Q, Lee CW, Morzycki W, Feld R, et al. Brief report: a phase II study of sunitinib in malignant pleural mesothelioma. the NCIC Clinical Trials Group. J Thorac Oncol 2011;6:1950-4

37. Song MS, Salmena L, Pandolfi PP. The functions and regulation of the PTEN tumour suppressor. Nat Rev Mol Cell Biol 2012;13:283-96

38. Loffler MW, Steinhilber J, Hilke FJ, Haen SP, Bosmuller H, Montes-Mojarro IA, et al. First case report of malignant peritoneal mesothelioma and oral verrucous carcinoma in a patient with a germline PTEN mutation: a combination of extremely rare diseases with probable further implications. BMC Med Genet 2018; $19: 144$

39. Ujiie H, Kadota K, Nitadori JI, Aerts JG, Woo KM, Sima CS, et al. The tumoral and stromal immune microenvironment in malignant pleural mesothelioma: A comprehensive analysis reveals prognostic immune markers. Oncoimmunology 2015;4:e1009285

40. Cornelissen R, Lievense LA, Maat AP, Hendriks RW, Hoogsteden HC, Bogers AJ, et al. Ratio of intratumoral macrophage phenotypes is a prognostic factor in epithelioid malignant pleural mesothelioma. PLoS One 2014;9:e106742

41. Thapa B, Salcedo A, Lin X, Walkiewicz M, Murone C, Ameratunga M, et al. The Immune Microenvironment, Genome-wide Copy Number Aberrations, and Survival in Mesothelioma. J Thorac Oncol 2017;12:850-9

42. Milella M, Falcone I, Conciatori F, Cesta Incani U, Del Curatolo A, Inzerilli N, et al. PTEN: Multiple Functions in Human Malignant Tumors. Front Oncol 2015;5:24

43. Zhong M, Clarke S, Vo BT, Khan SA. The essential role of Gialpha2 in prostate cancer cell migration. Mol Cancer Res 2012;10:1380-8

44. Fu X, Li Y, Alvero A, Li J, Wu Q, Xiao Q, et al. MicroRNA-222-3p/GNAI2/AKT axis inhibits epithelial ovarian cancer cell growth and associates with good overall survival. Oncotarget 2016; 7:80633-54

45. Ni J, Liu Q, Xie S, Carlson C, Von T, Vogel K, et al. Functional characterization of an isoformselective inhibitor of PI3K-p110beta as a potential anticancer agent. Cancer Discov;2:425-33

46. Weigelt B, Warne PH, Lambros MB, Reis-Filho JS, Downward J. PI3K pathway dependencies in endometrioid endometrial cancer cell lines. Clin Cancer Res;19:3533-44

47. Dolly SO, Wagner AJ, Bendell JC, Kindler HL, Krug LM, Seiwert TY, et al. Phase I Study of Apitolisib (GDC-0980), Dual Phosphatidylinositol-3-Kinase and Mammalian Target of Rapamycin Kinase Inhibitor, in Patients with Advanced Solid Tumors. Clin Cancer Res 2016;22:2874-84

48. Bedard PL, Tabernero J, Janku F, Wainberg ZA, Paz-Ares L, Vansteenkiste J, et al. A phase Ib dose-escalation study of the oral pan-PI3K inhibitor buparlisib (BKM120) in combination with 
the oral MEK1/2 inhibitor trametinib (GSK1120212) in patients with selected advanced solid tumors. Clin Cancer Res 2015;21:730-8

49. van Geel R, Tabernero J, Elez E, Bendell JC, Spreafico A, Schuler M, et al. A Phase Ib DoseEscalation Study of Encorafenib and Cetuximab with or without Alpelisib in Metastatic BRAFMutant Colorectal Cancer. Cancer Discov 2017;7:610-9

50. Bonnevaux H, Lemaitre O, Vincent L, Levit MN, Windenberger F, Halley F, et al. Concomitant Inhibition of PI3Kbeta and BRAF or MEK in PTEN-Deficient/BRAF-Mutant Melanoma Treatment: Preclinical Assessment of SAR260301 Oral PI3Kbeta-Selective Inhibitor. Mol Cancer Ther 2016; $15: 1460-71$ 


\section{Figure Legends}

Figure 1. Mesothelial-specific inactivation of Pten and Trp53 results in SMM development. A, Schematic representation of the conditional targeting of the Pik3 $\mathrm{ca}^{H 1047 R}$ mutation in the endogenous Pik3ca locus. B, H\&E staining of a mesothelioma from an adeno-Cre inoculated Pten;Trp53-null mouse growing extrinsically to the bladder wall. Scale bar, $200 \mu \mathrm{m}$ (left), $50 \mu \mathrm{m}$ (right). C, Adult Rosa26-LSLLacZ mice were inoculated with either PBS or adeno-Cre in the bladder and stained for $\beta$ Gal activity 2 weeks after injection. Scale bar, $200 \mu \mathrm{m}$ (left), $50 \mu \mathrm{m}$ (right). D, H\&E staining of a Pten;Trp53-null SMM

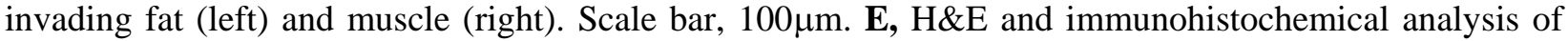
the expression of the indicated proteins in representative sections of tumors from Pten;Trp53-null and

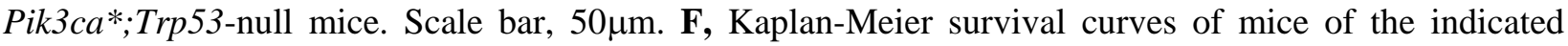
genotypes injected with adeno-Cre and monitored prospectively; $* * * P<0.001$, Log-rank (Mantel-Cox) test.

Figure 2. Pten; Trp53-null MMs exhibit an immunosuppressive stroma. A, Representative cleavedCaspase 3 and phospho-Histone 3 staining images of Pten;Trp53-null and Pik3ca*;Trp53-null tumors. Scale bar, $50 \mu \mathrm{m}$. B, C, Normalized DAB signal of staining of the indicated markers in Pten;Trp53-null and $P i k 3 \mathrm{Ca}^{*} ; \operatorname{Trp} 53$-null mouse tumors. Graphs show mean+SEM. $* P<0.05, * * P<0.01$, Mann-Whitney UTest. D, Representative images of immunohistochemical analysis of the indicated immune components in Pten;Trp53-null and Pik3ca*;Trp53-null tumors. Scale bar, 50 $\mu$ m. E, PD-L1 and Ppib RNAscope analysis in Pten;Trp53-null and Pik3ca*;Trp53-null MMs. Representative sections are shown. F, Graphs show mean+SEM of Ppib-normalized PD-L1 RNAscope signal in Pten;Trp53-null and Pik3ca*;Trp53-null tumors. $* P<0.05, * * P<0.01$, Mann-Whitney U-Test.

Figure 3. Human SMM tumor samples display low PTEN expression. A, Representative PTEN immunohistochemistry staining displaying strong (upper-left panel), moderate (upper-right panel), low (lower-right) and no staining (lower-left), Scale bar, $50 \mu \mathrm{m}$. B, Bar graph representing the proportion of cases scored as PTEN-positive and PTEN-negative tumors with epithelioid $(n=122)$, biphasic $(n=20)$, and sarcomatoid $(n=7)$ features. C, Box plot representing PTEN histoscores in the epithelioid $(n=122)$, biphasic $(\mathrm{n}=20)$ and sarcomatoid $(\mathrm{n}=7)$ tumor subgroups. ${ }^{*} P<0.05$, Tukey post-hoc test. D-E, Box plot representing indicated gene mRNA normalized counts in transcriptomically defined (D, upper panels) and histological subtypes (D, lower panels) from Bueno et al. (D) and TCGA (E) cohorts. ${ }^{*} P<0.05, * * P<0.01$, $* * * P<0.001$ Tukey post-hoc test. 
Figure 4. Pten inactivation results in tumors with strong Mek/Erk activation, involved in tumor cell proliferation and in vitro invasiveness. A, B, WB analysis of the indicated proteins using lysates of tumors from adeno-Cre inoculated Pten;Trp53-null and Pik3ca*;Trp53-null mice. C, Densitometric quantitation of signal intensity of phosphorylated proteins normalized to total protein (pAkt/Akt, pErk1/2 / Erk1/2, pMek1/2 / Mek1/2) using ImageJ software. Graphs show mean \pm SEM; $* P<0.05$, ** $P<0.01$, MannWhitney U-Test. D, Phase contrast images of cultures established from Pten;Trp53-null and Pik3ca*;Trp53-null tumors. E, WB analysis from a representative experiment $(\mathrm{n}=4)$ of the indicated proteins using total lysates of exponentially growing Pten;Trp53-null and Pik3ca*;Trp53-null MM cells. F, Effects of PD325901 $(0.5 \mu \mathrm{M})$ on the proliferation of Pten;Trp53-null and Pik3ca*;Trp53-null MM cells. Graphs show mean \pm SEM of fold-increase in cell number with respect to the $0 \mathrm{~h}$ time point $(\mathrm{n}=4$ independent experiments). ${ }^{*} P<0.05$, Mann-Whitney U-Test. G, Effects of PD325901 (0.5 $\left.\mu \mathrm{M}\right)$ on the in vitro invasion of Pten;Trp53-null and Pik3ca*;Trp53-null MM cells assessed after $48 \mathrm{~h}$. Invading and non-invading cells were visualized by confocal microscopy. Graphs show mean \pm SEM of the $\%$ of invading cells per field ( $\mathrm{n}=4$ independent experiments). $* P<0.05$, Mann-Whitney U-Test.

Figure 5. Gai2 signaling mediates pErk and pAkt levels in Pten;Trp53-null MM. A, Phospho-RTK dot blot array analysis using lysates of tumors from adeno-Cre inoculated Pten;Trp53-null and Pik3ca*;Trp53-null mice. Phospho-receptors showing differences in signal intensity between both genotypes are highlighted: \#1, Erbb4; \#2, Fgfr3; \#3, Scfr; \#4, Flt-3; \#5, EphB1. B, The heatmap shows ImageJ software signal quantification of all the RTK included in the array. C, Effects of PTX 100ng/ml (15h) on signaling in Pten;Trp53-null and Pik3ca*;Trp53-null MM cells. Cell lysates were analyzed by WB with the indicated antibodies. Representative results of $\geq 3$ independent experiments. D, Boxplots of $\log 10$-transformed normalized count values for each Gai gene in Pten;Trp53-null and Pik3ca*;Trp53-null tumors. E, Immunohistochemical analysis of Gai2 expression in representative sections of tumors from

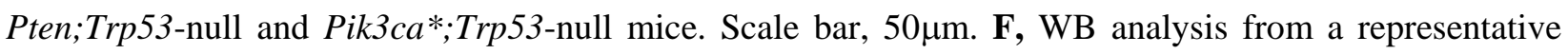
experiment $(\mathrm{n}=2)$ of the indicated proteins using total lysates of exponentially growing Pten;Trp53-null and Pik3ca*;Trp53-null MM cells infected either with non-targeting (Nt) or Gai2-targeting shRNAs. G, Box plot representing GNAI2 RNAseq normalized counts of transcriptomically defined (left) and histological (right) subtypes from Bueno et al. sample cohort.

Figure 6. Selumetinib and AZD8186 synergize to impair the in vivo growth of Pten;Trp53-null MM. A, Effects of PD325901 $(0.5 \mu \mathrm{M})$, TGX-221 $(10 \mu \mathrm{M})$, and their combination (PD+TGX) on the 
proliferation of Pten;Trp53-null MM cells. Graphs show mean \pm SEM of fold-increase with respect to the $0 \mathrm{~h}$ time point ( $\mathrm{n}=3-5$ independent experiments). $\mathrm{P}^{*}<0.05$, Mann-Whitney U-Test. B, Effects of PD325901 $(0.5 \mu \mathrm{M})$, TGX-221 $(10 \mu \mathrm{M})$, and their combination (PD+TGX) on the in vitro invasion of Pten;Trp53-null MM cells assessed at 24h. Graphs show mean \pm SEM of the $\%$ of invading cells per field in the different conditions ( $\mathrm{n}=4$ independent experiments). ${ }^{*} P<0.05, * * P<0.01$, Mann-Whitney U-Test. $\mathbf{C}$, Representative H\&E stainings of Pten;Trp53-null SMM displaying bone differentiation (left panel, Scale

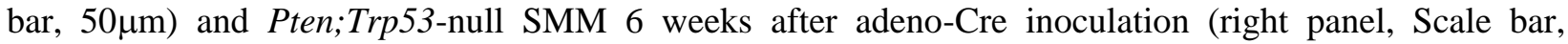
$200 \mu \mathrm{m})$. D, Kaplan-Meier survival curves of Pten;Trp53-null tumor-bearing mice treated for 7 weeks with vehicle, Selumetinib (10mg/kg), AZD8186 (40mg/kg) or both (S+AZD) (n=6/group). $* P<0.05$, Logrank (Mantel-Cox) test. E, Representative axial micro-CT images of the abdomen of adeno-cre inoculated Pten;Trp53-null mice before and 1-3 weeks after treatment. Arrows indicate the bladder; tumors (with bone differentiation signal) are indicated by an asterisk. Thickening of the abdominal wall is shown as an arrowhead. F, Kaplan-Meier survival curves of Pten;Trp53-null tumor-bearing mice treated for 12 weeks with vehicle, or both (S+AZD, 10mg/kg Selumetinib, 40mg/kg AZD8186) (n=11/group). **P<0.01, Logrank (Mantel-Cox) test.

Figure 7. The Selumetinib/AZD8186 combination inhibits proliferation of primary human cultures derived from non-epithelioid human pl-MM harboring TP53 mutations. A, Graphs show \% cell viability \pm SEM $(n=2-3)$ after $72 \mathrm{~h}$ of exposure to serial drug dilutions or to the drug combination (S+AZD) at a constant ratio for Selumetinib/AZD8186 responder cells. Compound concentrations range from 0.025 to $20 \mu \mathrm{M}$. The combination ratios for Selumetinib and AZD8186 used for each specific cell line are specified in Supplementary Table S3. B, The heatmap shows normalized AUC values for serial dilutions of the indicated compounds, representing drug response of each human MPM primary culture. Original tumor histology of the cell lines is indicated. C, Normalized AUC for Selumetinib, AZD8186 or the combination (S+AZD), for cultures derived from EMM, BMM, and SMM. $* P<0.05$, Mann-Whitney U-Test. D, WB analysis of the indicated proteins using lysates of primary human MM cultures. Original tumor histology of the cell lines, TP53 status and normalized AUC are also indicated E, The heatmap represents unsupervised clusterization of human primary MPM cells based on densitometric ImageJ software quantitation of WB signal intensities of Gai2, Vimentin, and AKT1/2 proteins normalized to Vinculin. Normalized AUC values of each cell line are also indicated. F, GI50 values for cisplatin and for Selumetinib/AZD8186 (S+AZD) for combination-responder cells and combination non-responder cells. 
A

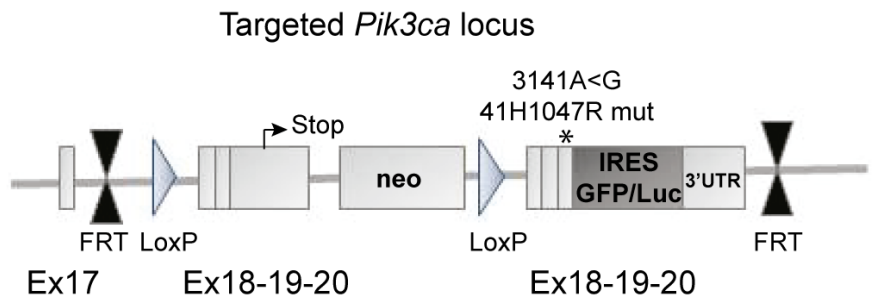

C

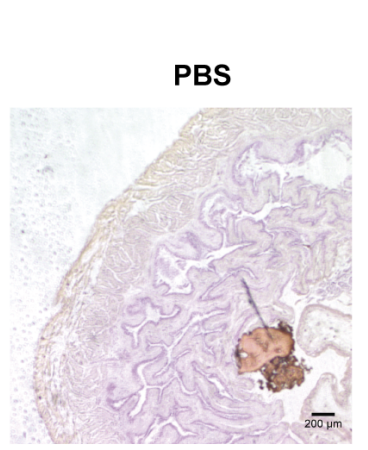

Rosa26-LSL- $\beta$ Gal
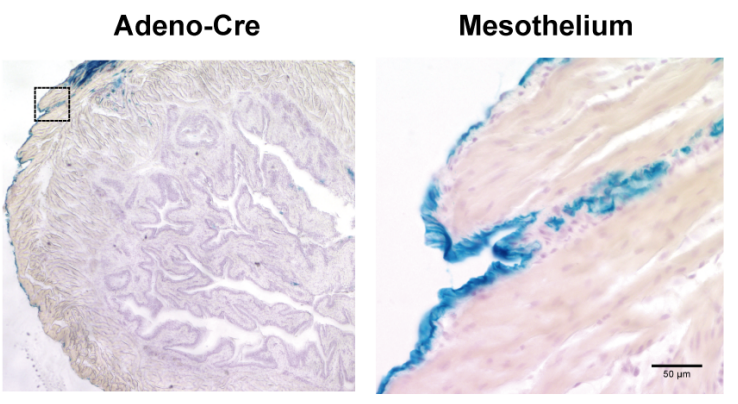

E

H\&E
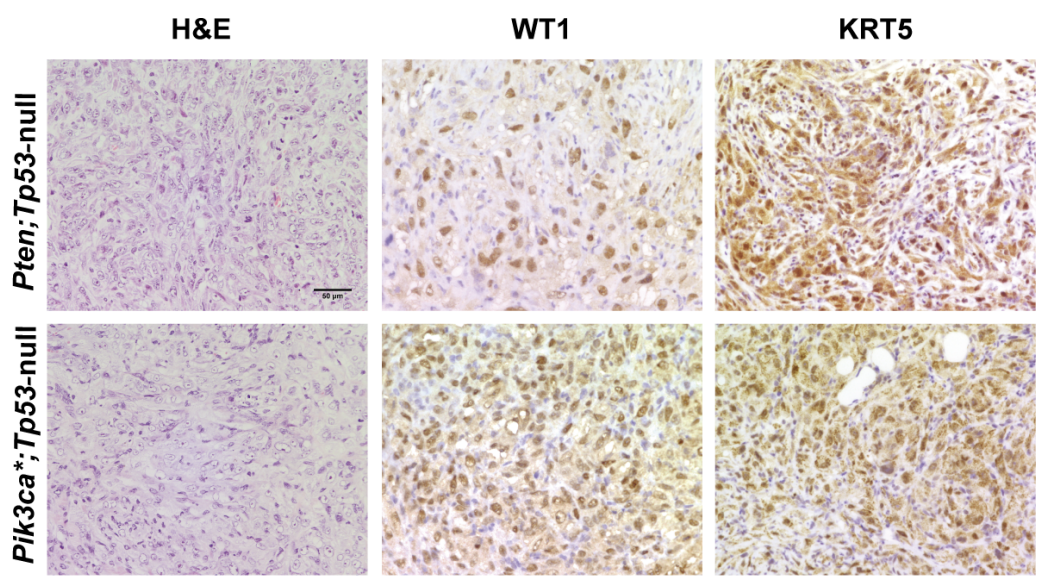
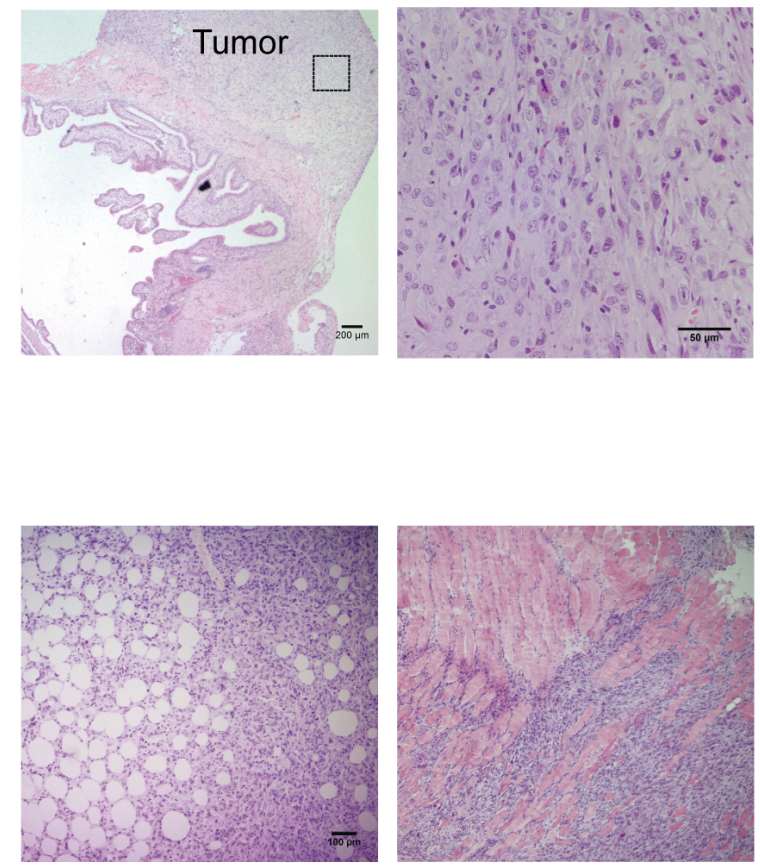

D

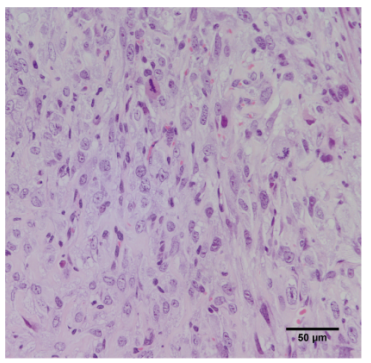

F

Vimentin

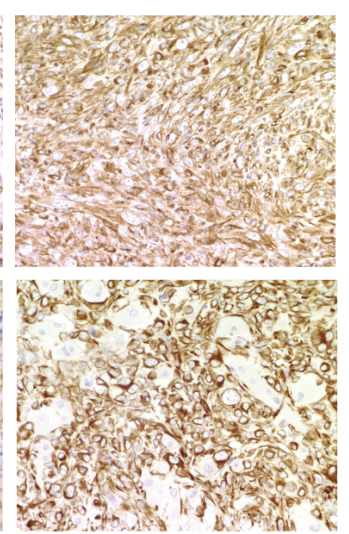

$\rightarrow$ Trp53-null $(\mathrm{n}=11)$

$\rightarrow$ Pten;Trp53-null $(\mathrm{n}=10)$

- Pik3ca*;Trp53-null ( $\mathrm{n}=15)$

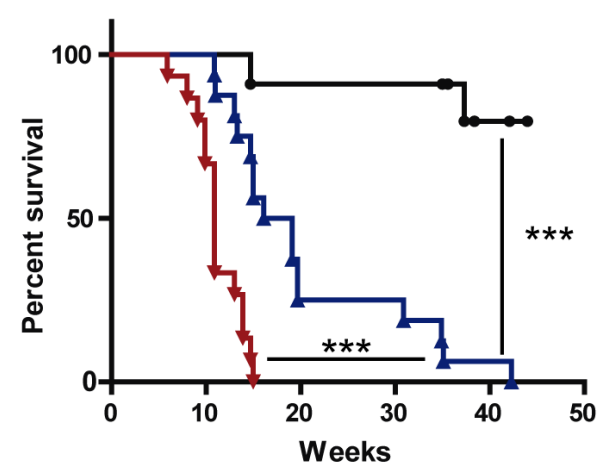


A

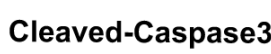

D

F4/80

Ym1
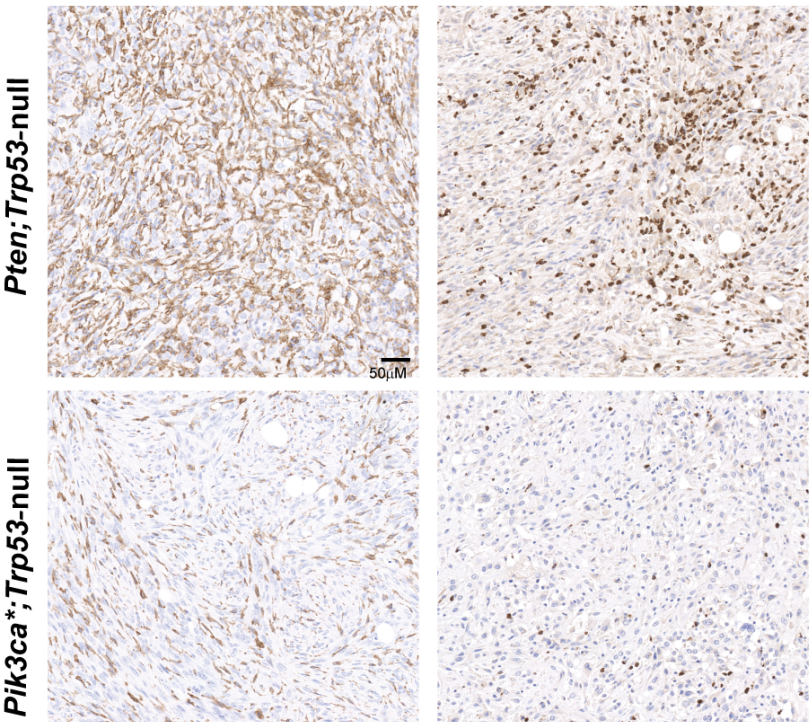

$\overline{50 \mu \mathrm{M}}$

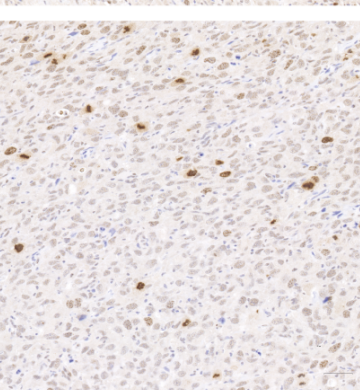

B
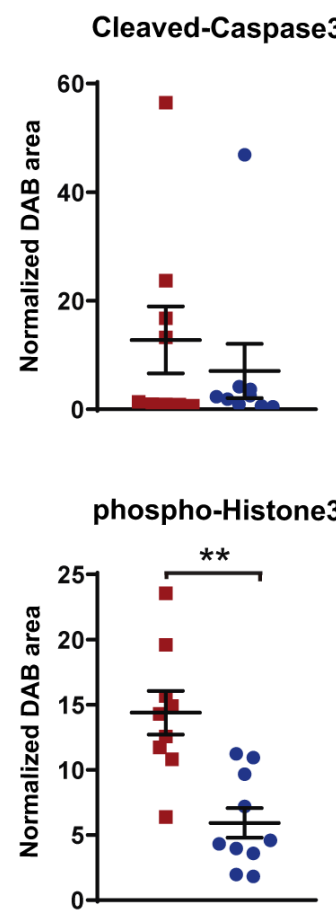

Cd3
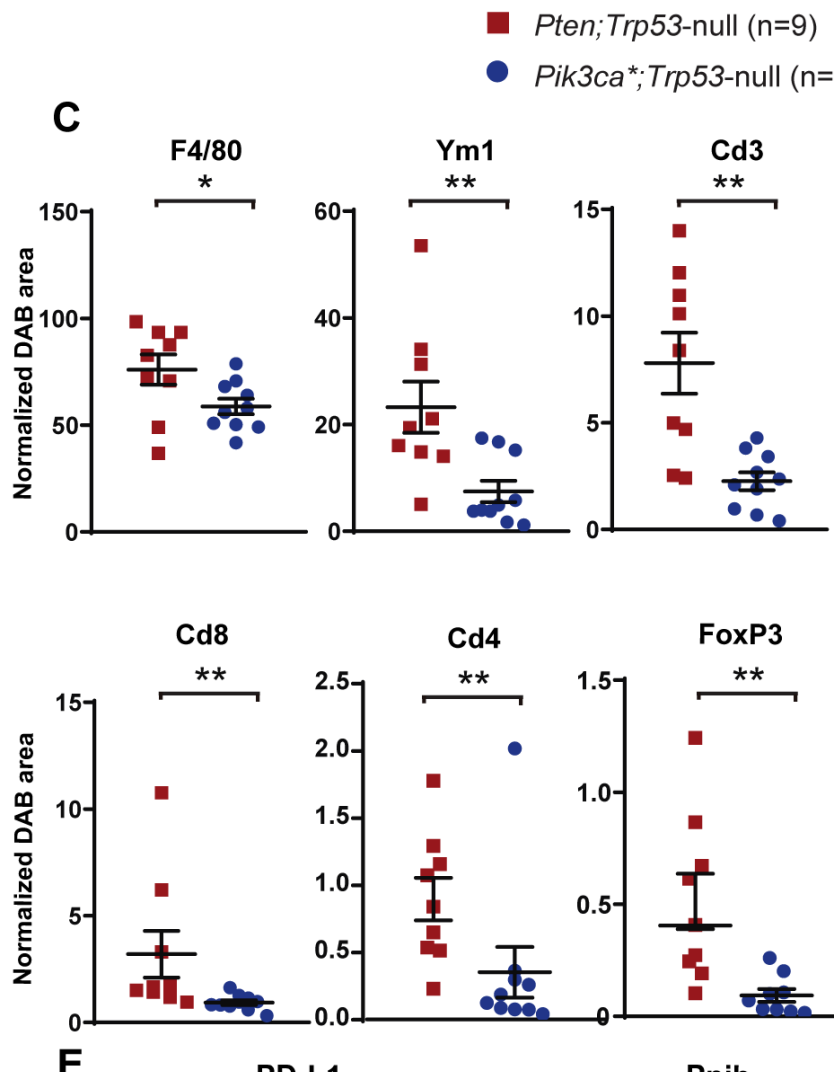

PD-L

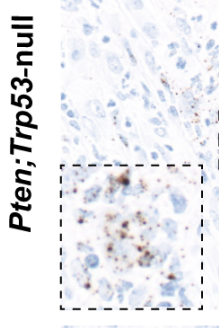

है

FoxP3
F

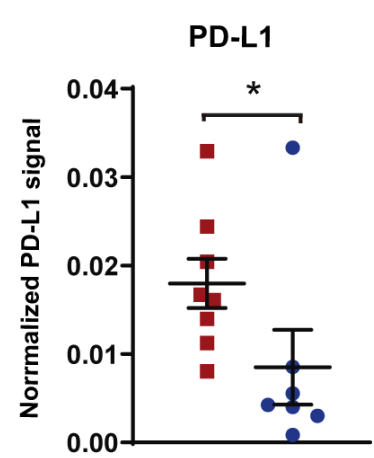


B
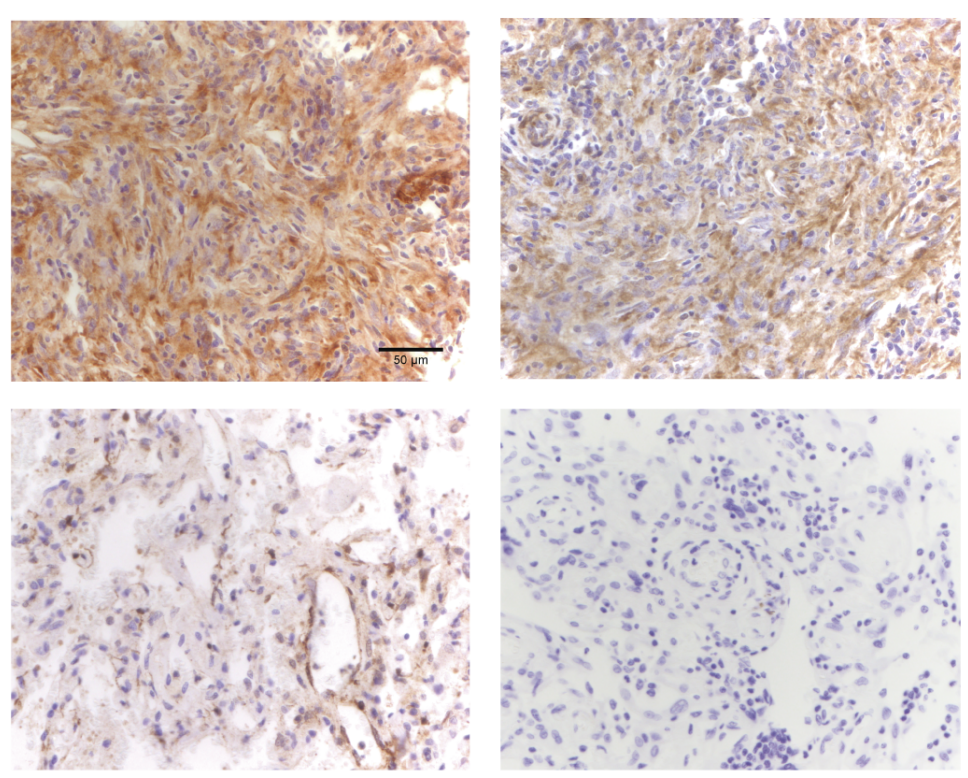
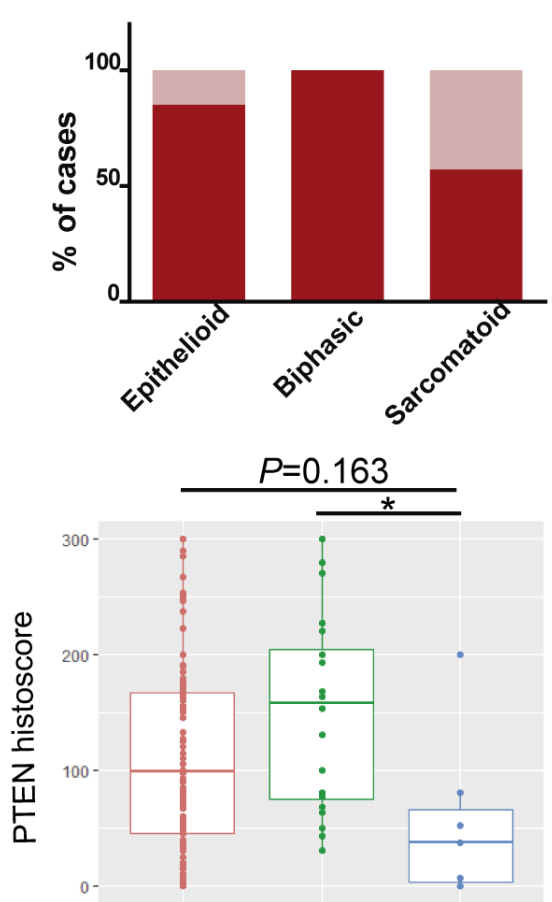

Epithelioid Biphasic Sarcomatoid
PTEN negative

PTEN positive
D
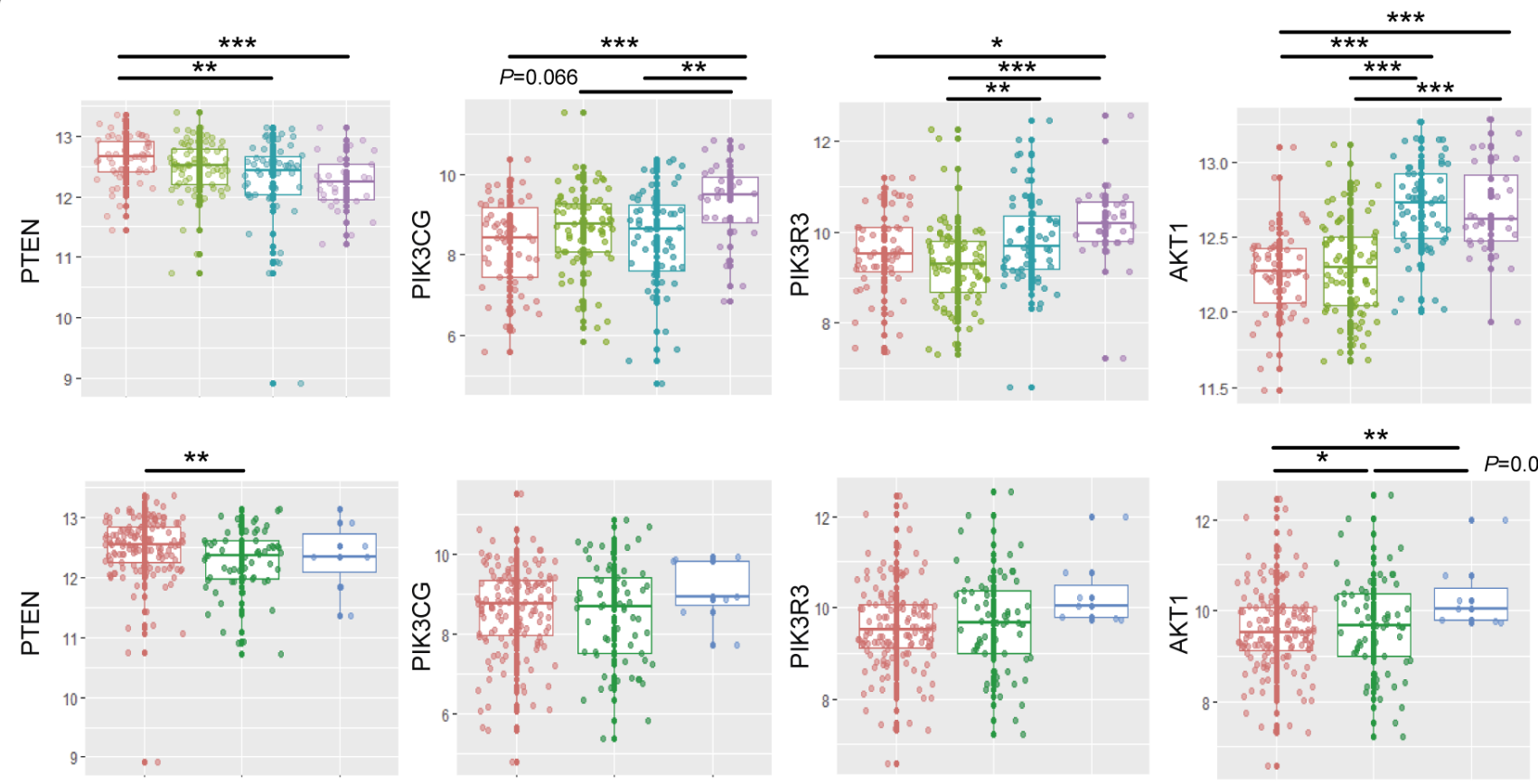

E
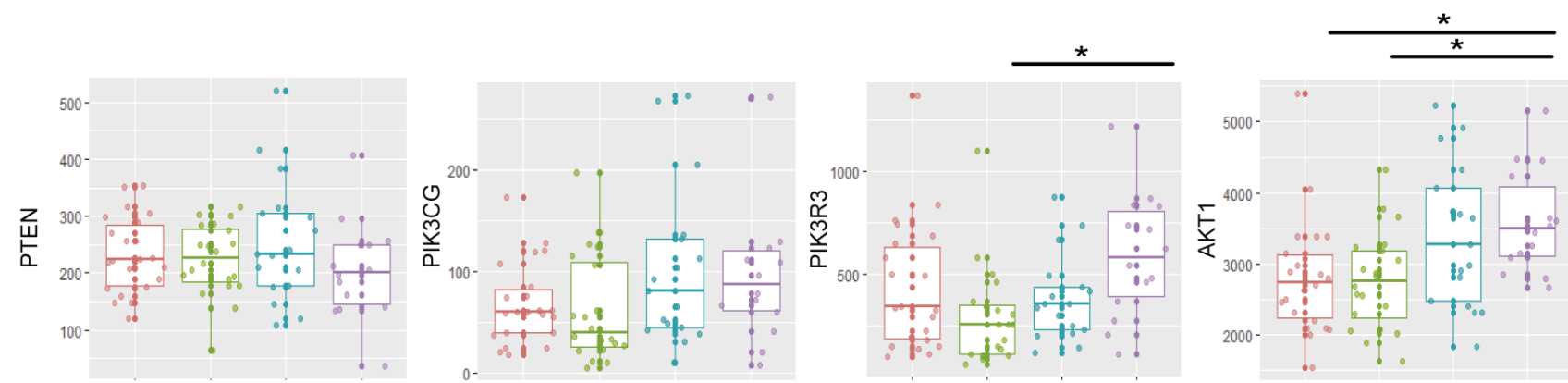

Epithelioid

'b Biphasic-E

Biphasic-S

Sarcomatoid

Epithelioid

$n=209$

Epithelioid
Biphasic-E 
A

Pten;Trp53-null Pik3ca*;Trp53-null

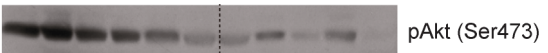

$\begin{array}{l:ll}--1 & \text { pAkt (Thr308) }\end{array}$

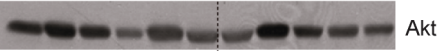

료 $=\quad$ pErk1/2

- -1 (Thr202/Tyr204)

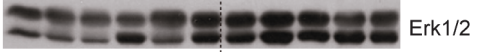

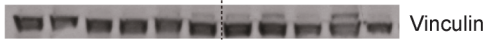

B

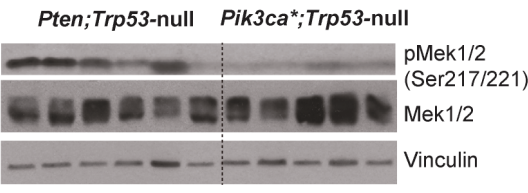

C

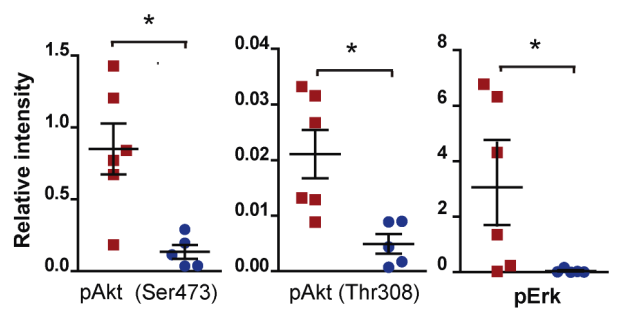

- Pik3ca*;Trp53-null $(\mathrm{n}=5)$

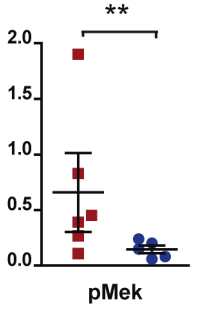

D

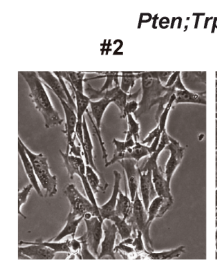

\#3

P1 Pik3ca*;Trp53-null
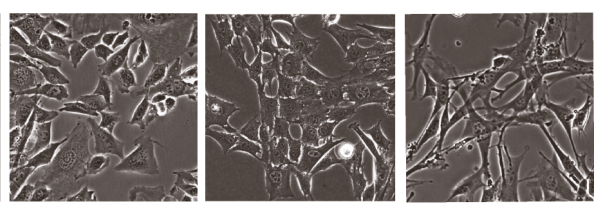

E
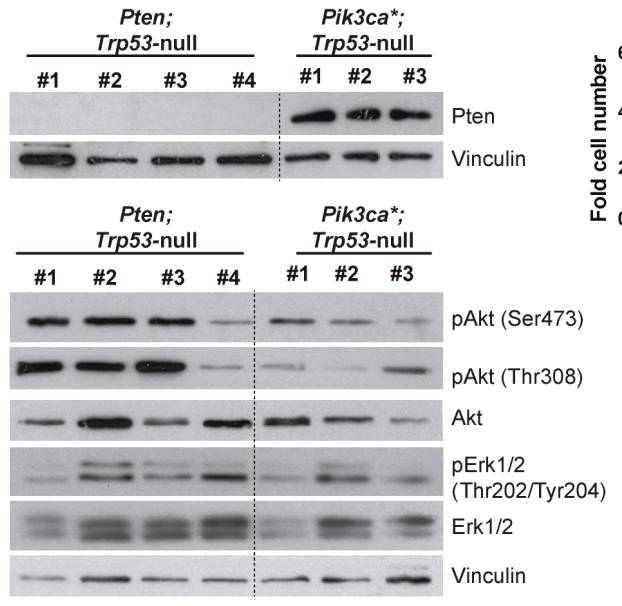

F
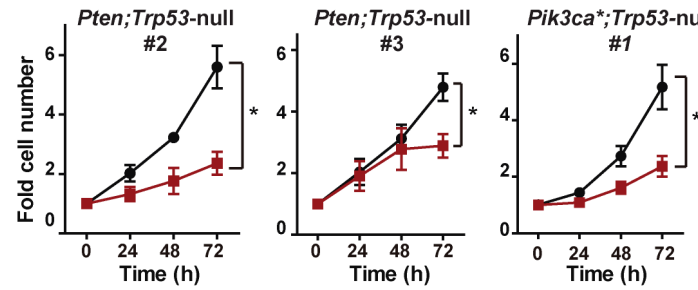

- Vehicle

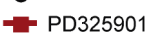

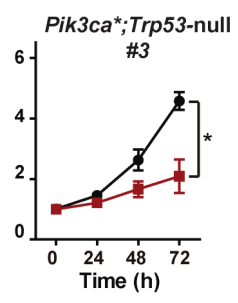

G

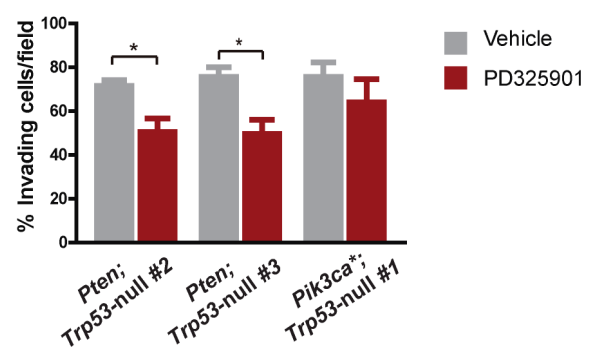


A

\section{Pten;Trp53-null}

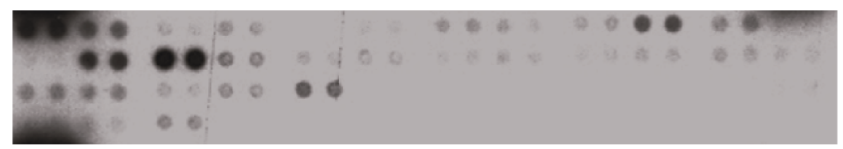

Pik3ca*;Trp53-null

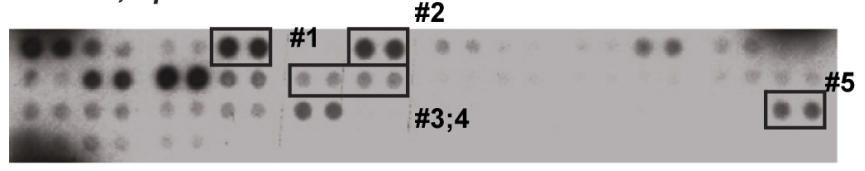

B

\section{Relative intensity}

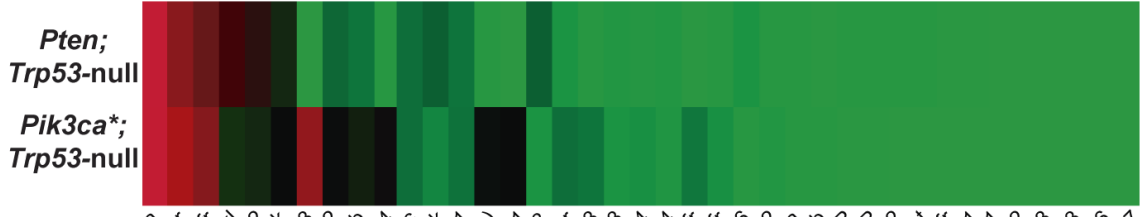

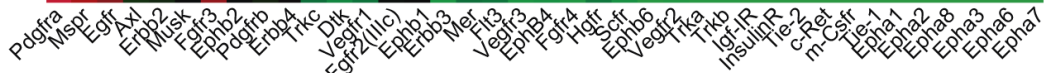

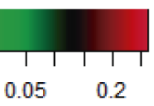

D Value

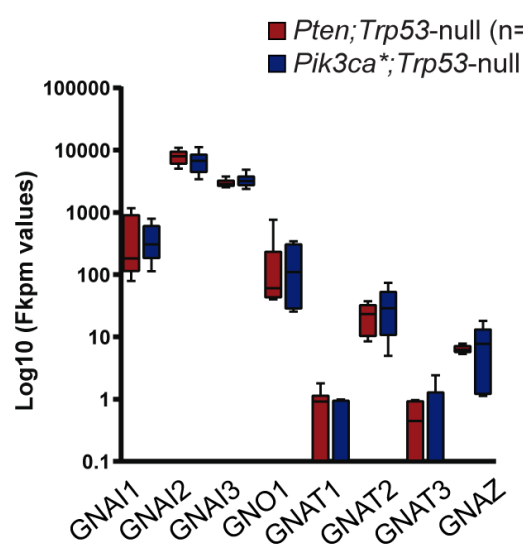

E

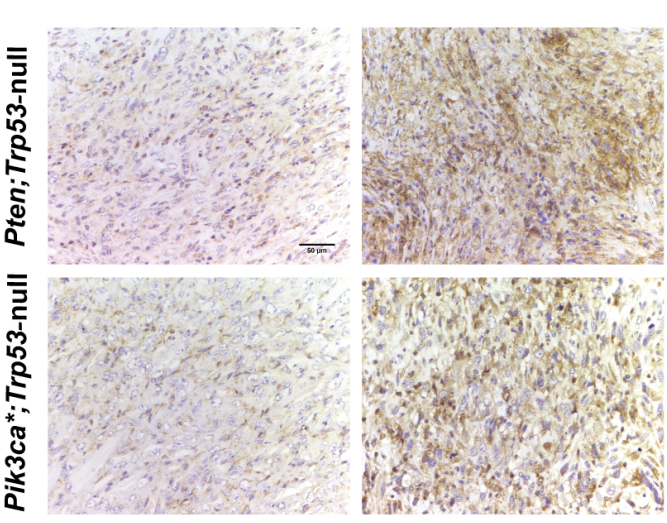

$\mathbf{F}$

Pten;Trp53-null Pik3ca*;Trp53-null

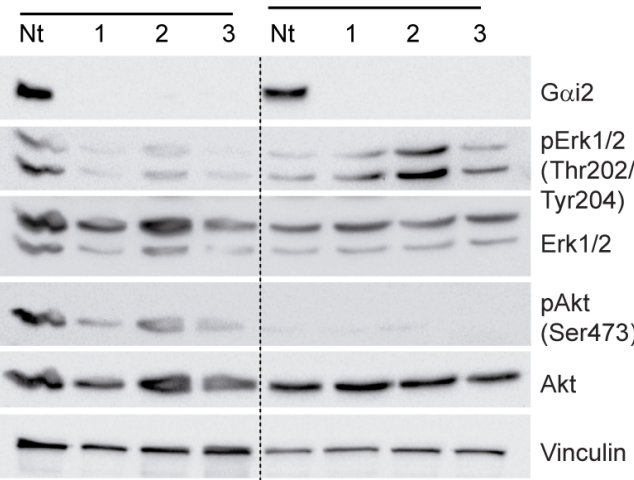

G

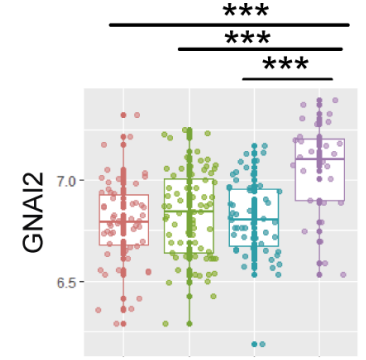

Epithelioid

Biphasic-E

Biphasic-S

Sarcomatoid

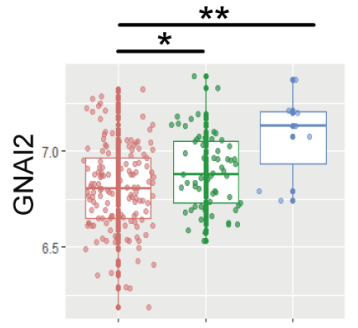

Epithelioid

Biphasic

Sarcomatoid 
A

Pten;Trp53-null \#2

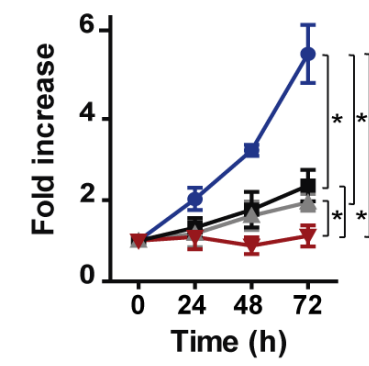

Time (h)
Pten;Trp53-null \#3

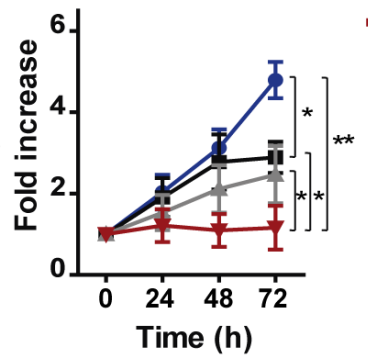

B

- Vehicle

$\Rightarrow$ TGX-221

$=$ PD+TGX

C

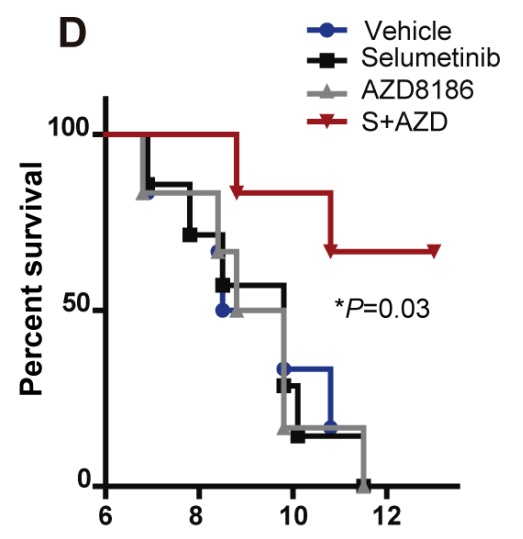

Weeks after adeno inoculation
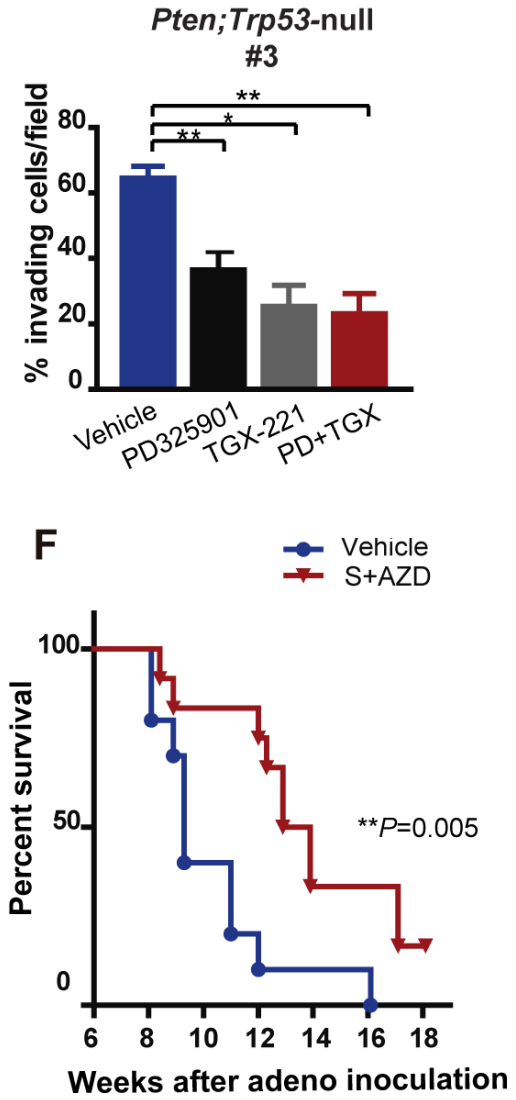

E

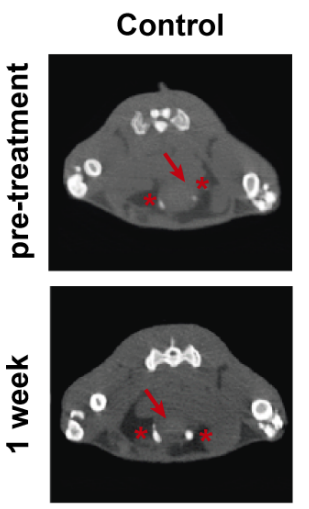

Selumetinib

AZD8186
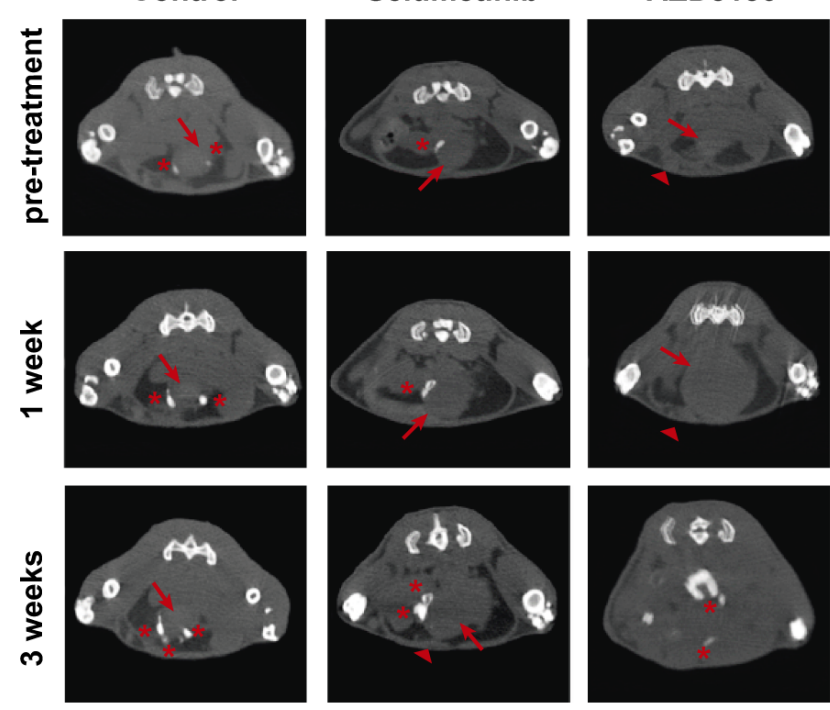

S+AZD
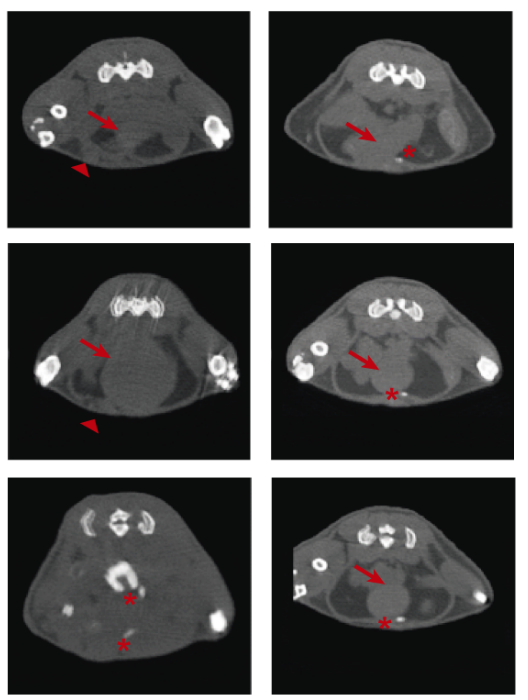
A
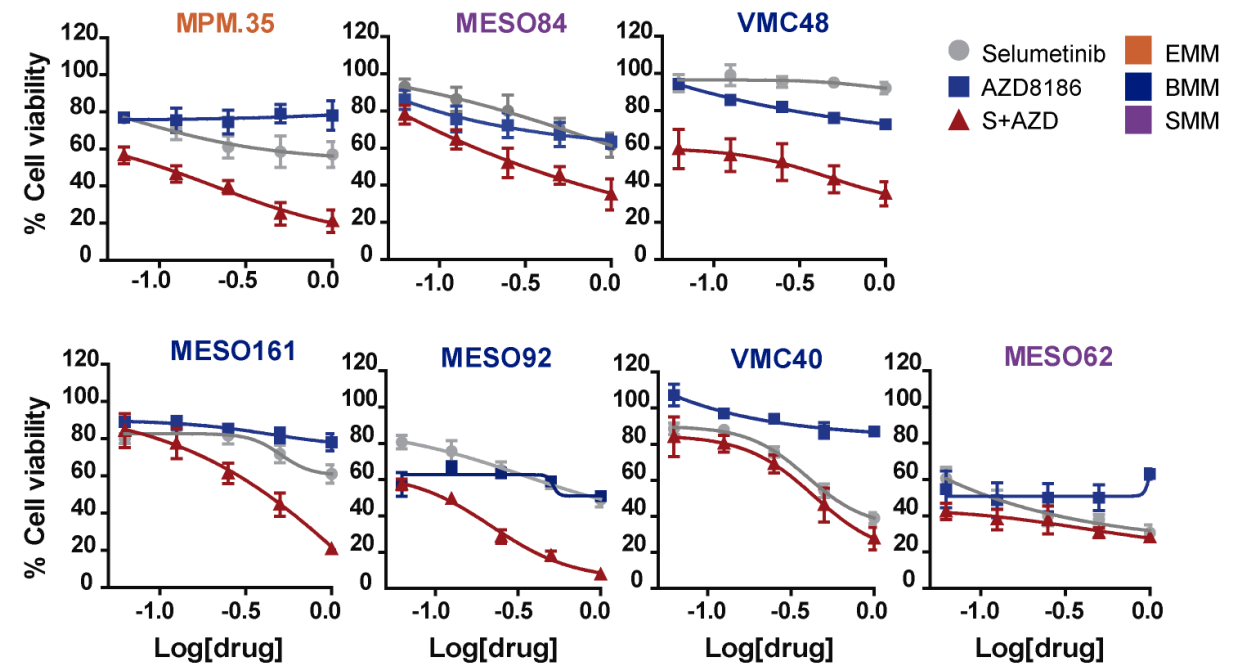

c

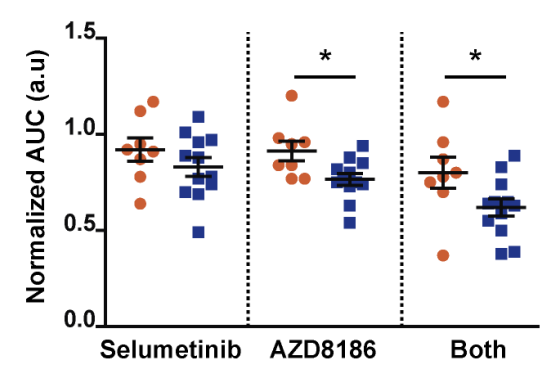

E

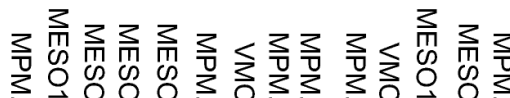

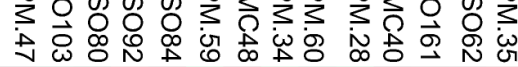

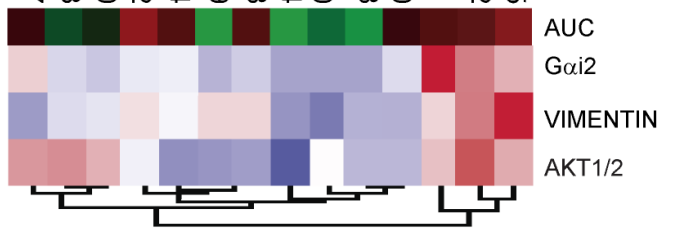

Relative protein expression

$\begin{array}{lllll}2 & 1 & 0 & -1 & -2\end{array}$
B

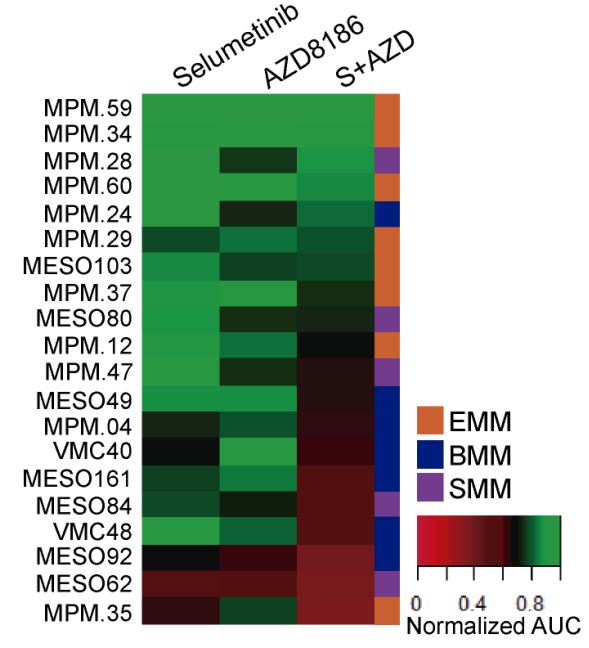

Tumor histology TP53 status
EMM $\quad$ wt
BMM mut/null
SMM

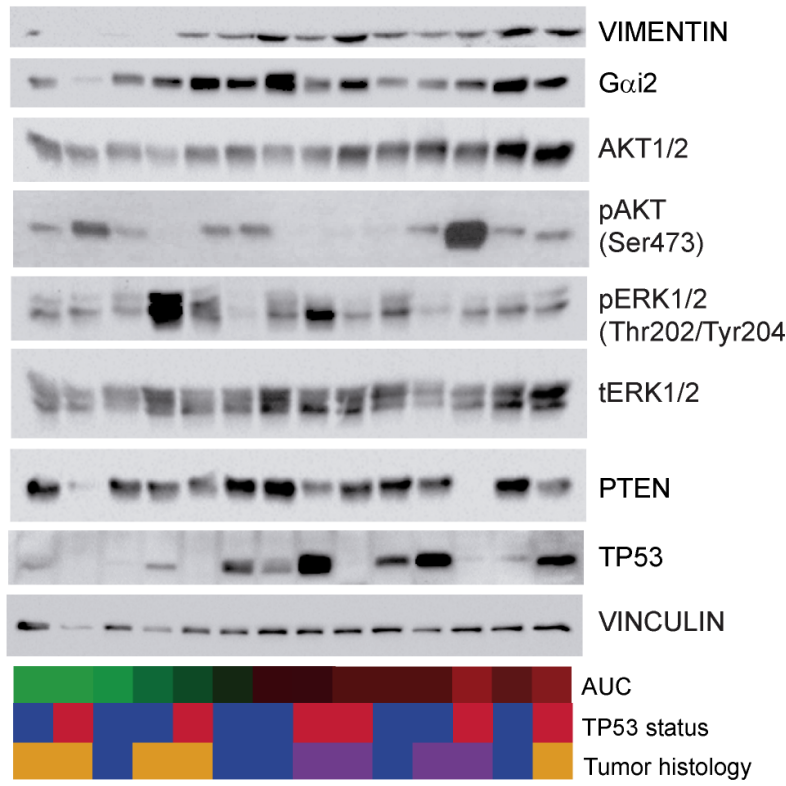

Normalized AUC

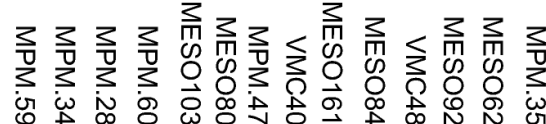

$$
\text { ) }
$$

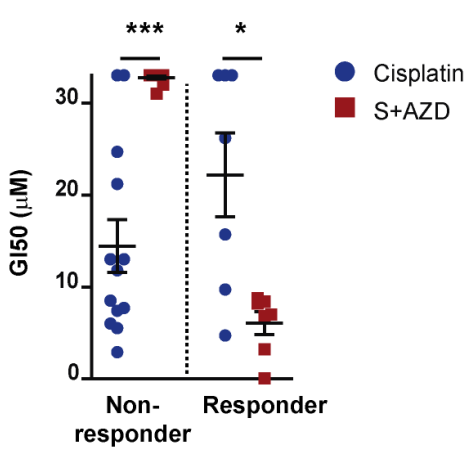

